University of South Florida

DIGITAL COMMONS

Digital Commons @ University of

@ UNIVERSITY OF SOUTH FLORIDA

South Florida

USF Tampa Graduate Theses and Dissertations

USF Graduate Theses and Dissertations

3-23-2017

\title{
Failure Analysis of High Nickel Alloy Steel Seal Ring Used in Turbomachinery
}

Wenbo Wang

University of South Florida, wenbowang@mail.usf.edu

Follow this and additional works at: https://digitalcommons.usf.edu/etd

Part of the Engineering Commons

\section{Scholar Commons Citation}

Wang, Wenbo, "Failure Analysis of High Nickel Alloy Steel Seal Ring Used in Turbomachinery" (2017). USF Tampa Graduate Theses and Dissertations.

https://digitalcommons.usf.edu/etd/6635

This Thesis is brought to you for free and open access by the USF Graduate Theses and Dissertations at Digital Commons @ University of South Florida. It has been accepted for inclusion in USF Tampa Graduate Theses and Dissertations by an authorized administrator of Digital Commons @ University of South Florida. For more information, please contact digitalcommons@usf.edu. 
Failure Analysis of High Nickel Alloy Steel Seal Ring Used in

Turbomachinery

by

Wenbo Wang

\begin{abstract}
A thesis submitted in partial fulfillment
of the requirements for the degree of

Master of Science in Materials Science and Engineering

Department of Mechanical Engineering

College of Engineering

University of South Florida
\end{abstract}

Major Professor: Alex Volinsky, Ph.D.

Wenjun Cai, Ph.D.

Venkat R. Bhethanabotla, Ph.D.

Date of Approval:

March 9, 2017

Keywords: Recrystallization, Precipitates, Grain refinement, Hardness, In-situ tensile test Copyright (C) 2017, Wenbo Wang 


\section{DEDICATION}

I highly appreciate my family for always selflessly supporting and understanding me. To my advisor Prof. Alex Volinsky, who teaches me a lot in academy and guides me to finish my thesis.

To my dear friends, who inspire me for my life and study. 


\section{ACKNOWLEDGMENTS}

This research was supported by the National Natural Science Foundation of China (51271022, 51431004), Beijing Higher Education Young Elite Teacher Project (YETP0353), National Basic Research Program of China (2012CB937502) and by the National Science Foundation (IRES 1358088).

I would like to thank my advisor Prof. Alex Volinsky. He always has enough patience to help me when I met some academic problems. Moreover, I am grateful to the Pro. Xiaolu Pang who is at the University of Science and Technology Beijing and his student Chengzhi Zheng. They gave my a great support in my experiments. 


\section{TABLE OF CONTENTS}

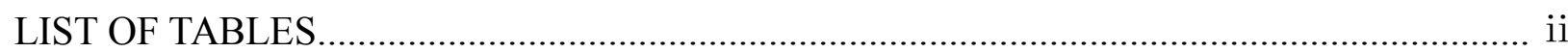

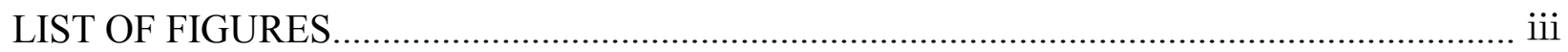

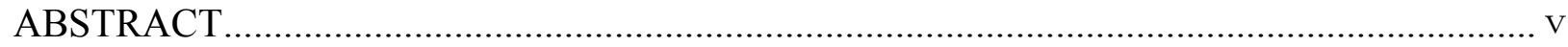

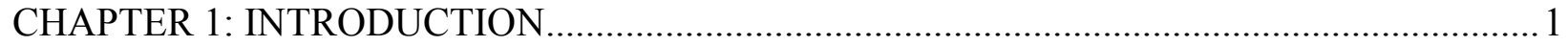

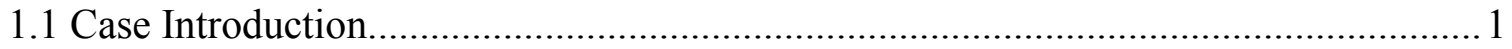

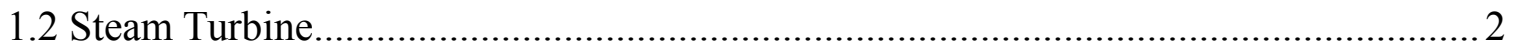

1.2.1 Steam Turbine Basic Components............................................................ 4

1.3 The Principle of Seal...........................................................................................5

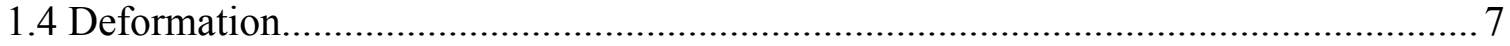

1.4.1 The Effect of Plastic Deformation on Organization of Metal.........................9

1.4.2 The Effect of Plastic Deformation on Properties of Metal........................... 10

1.5 Common Failure of Materials.................................................................................... 14

1.5.1 Material Safety Design Criterion and Two Types of Fracture....................... 15

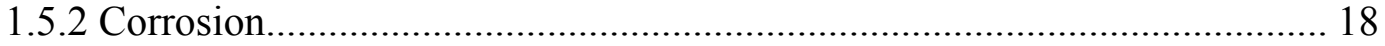

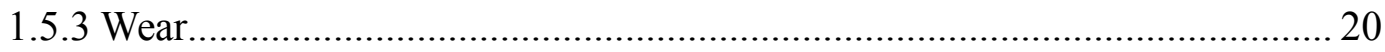

CHAPTER 2: MATERIAL MEASUREMENT METHODS....................................................... 23

2.1 Measurement at Macroscopic Scale and Specimen Preparation.................................. 23

2.2 The Hardness and Elastic Modulus of Seal Ring Test...............................................2 24

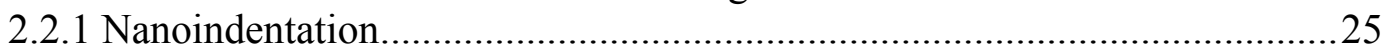

2.3 Scanning Electron Microscope and Energy-dispersive Spectrometer......................... 27

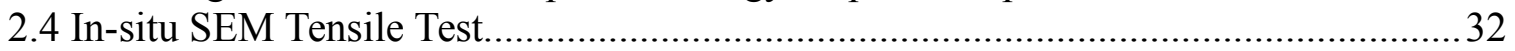

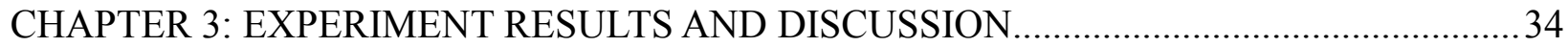

3.1 Macroscopic Dimensions Examination........................................................................ 34

3.2 Relationship Between the Opening Gap Length and the Applied Load....................... 35

3.3 Elastic Modulus, Hardness and Bulk Chemical Composition.........................................37

3.4 Metallographic, SEM and EDS Analyses................................................................ 38

3.5 In-situ SEM Tensile Test...................................................................................42

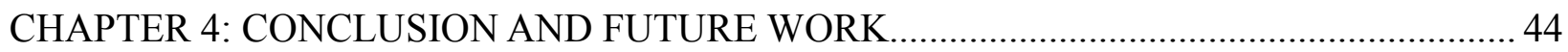

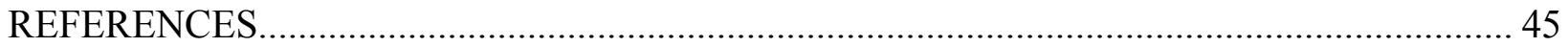

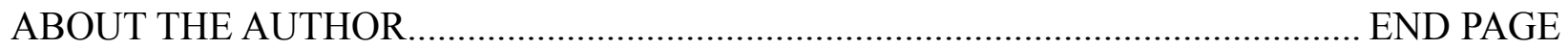




\section{LIST OF TABLES}

Table 1. Three common types of Rockwell hardness values................................................. 25

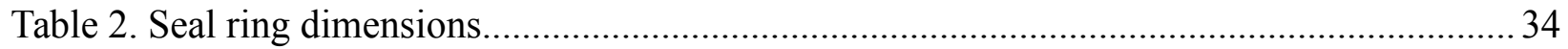

Table 3. Relationship between the externally applied load and

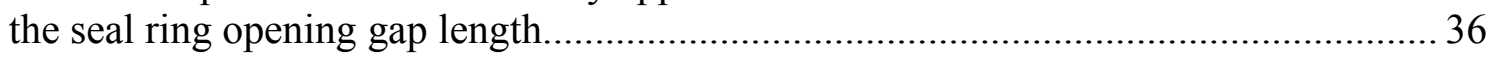

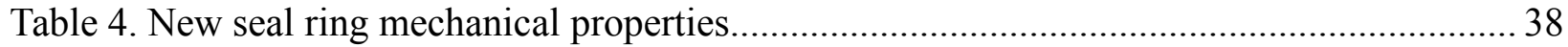

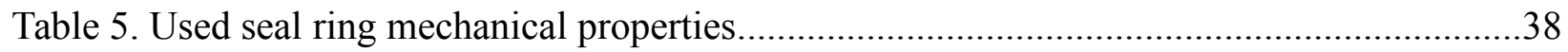

Table 6. Main elements composition of the used and new seal rings.................................... 38

Table 7. Used seal ring precipitate composition elements analysis by EDS............................ 40

Table 8. New seal ring precipitate composition elements analysis by EDS........................... 41 


\section{LIST OF FIGURES}

Figure 1. Schematics of the seal rings in the nuclear power turbine system................................ 2

Figure 2. Schematics of two types of turbines:

(a) Reaction turbine; (b) Impulse turbine.................................................................. 3

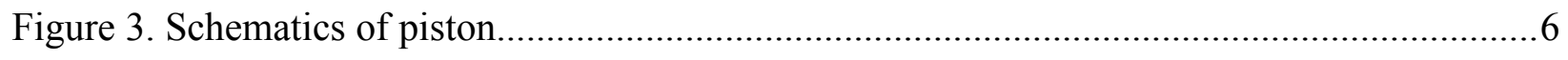

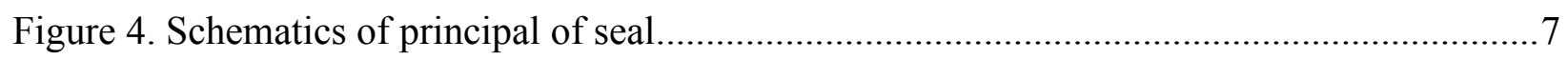

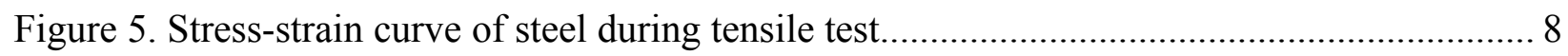

Figure 6. Schematics of fiber texture and sheet texture.........................................................10

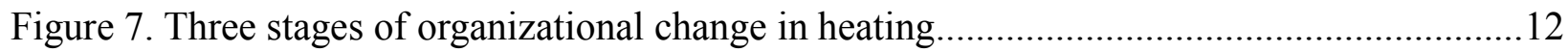

Figure 8. Schematics of dislocation arrangement:

(a) Before polygonization; (b) After polygonization................................................... 12

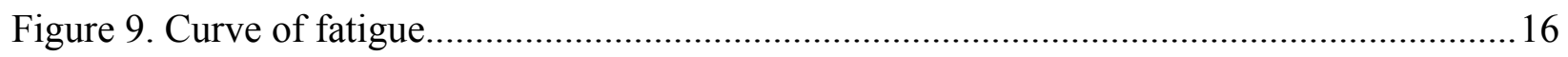

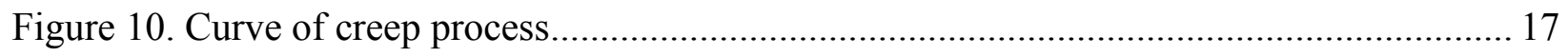

Figure 11. Schematics of adhesive wear between piston ring and cylinder wall..........................21

Figure 12. Specimens cut from: (a) New seal ring; (b) Used seal ring.......................................23

Figure 13. Schematics of nanoindentation technique: (a) Indenter at surface;

(b) Curve of load-displacement........................................................................... 26

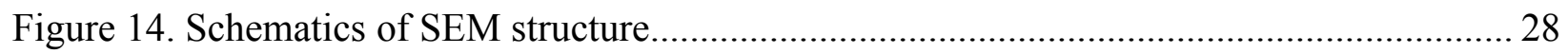

Figure 15. Interaction between electron beam and sample above the surface..............................29

Figure 16. Relationship between depth of field, aperture diameter

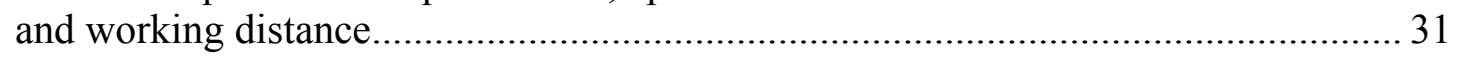

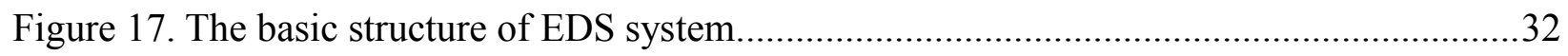

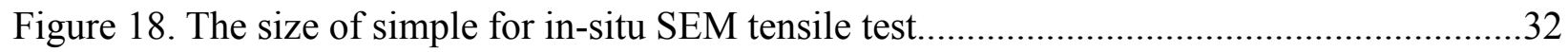


Figure 19. (a) Upper and lower seal rings on the turbine;

(b) Macroscopic ring dimensions measurements

Figure 20. Schematics of the opening gap length measurement method.....................................35

Figure 21. Relationships between the opening gap length and the externally applied load. .36

Figure 22. Optical microscopy images of: (a) New seal ring back surface;

(b) Used seal ring back surface showing precipitates along the grain boundaries.

Figure 23. (a) SEM micrograph; (b) Combined elemental map;

(c) Individual elemental maps; (d) EDS spectra of point 1 and

(e) EDS spectra of point 2 of the used seal ring labeled in (a)

Figure 24. SEM images of: (a) Used seal ring and (b) New seal ring obtained during in-situ tensile test 


\begin{abstract}
The system of upper high nickel alloying steel seal ring and lower high nickel alloying steel seal ring, installed in the grooves of turbine, can extend out and fit with the wall of valve cage, resulting in forming a good seal under the pressure. In the project, the failure steel seal ring is considered. This situation had threatened the safety of the whole steam turbine system. The purpose of this study is to identify the failure cause of the steel seal ring used in nuclear steam turbines. New high nickel steel alloy seal ring was compared with the failed seal ring.

The dimensions of macroscopic ring with clearly plastic deformation were measured using calipers. Surface morphology of ring was observed by optical microscopy through metallographic analysis. There is a lot of precipitation in the grain boundaries of used seal ring, along with smaller grain size than the new seal ring. To explore the composition of precipitation, scanning electron microscopy (SEM) with energy-dispersive spectrometer (EDS) were used. The results indicated that the concentration of titanium (Ti) and molybdenum (Mo) was higher in the precipitation of used seal ring. At the same time, the hardness and elastic modulus of used seal ring were reduced, measured by nanoindentation test. In-situ SEM tensile testing were used to record and analyze the generation of crack source and crack development under applied load. The reasons of the seal ring failure can be answered because of these experimental results at both macroscopic and microscopic scales.
\end{abstract}

The main reason of the seal ring failure is a combination of long-term stress and elevated temperature during turbine operation. Complex work environment caused recrystallization and 
recovery, resulting in grain refinement and secondary phase precipitation. Further embodiment, recrystallization and recovery caused the elastic modulus and hardness of used seal ring decrease. Moreover, a lot of secondary phase precipitates appeared at grain boundaries during use. The appearance of secondary phase precipitates become the weakest part of used seal ring. The applied load lead to seal ring failure from the formation of microvoids to microvoids aggregated becoming microcracks until to the appearance of cracks at macroscopic scale. These changes of microscopic structure ultimately reflected in critical plastic deformation of used seal ring. 


\section{CHAPTER 1: INTRODUCTION}

\subsection{Case Introduction}

Seal ring is one of the important parts of power steam turbines, which is an elastic metal ring operating on the principle of outward expansion. Gas or liquid pressure is needed to form a good seal, as shown schematically in Figure 1 [1]. The seal ring performance directly affects the safety and proper operation of the whole steam turbine unit. During operation, seal ring can be damaged due to long-term cyclic deformation, finally causing leakage. This thesis describes examples of the failure of the seal ring used in nuclear power turbines. The seal ring is made

from the high nickel alloy steel, grade GH163 (YB/T 5351-2006 Chinese standard) [2]. The ring failure was manifested by high-frequency metal noise coming from the working nuclear power turbine. When the used free-standing seal ring was hit, similar crisp metal sound could be heard. Inspection revealed insufficient tension of the seal ring, associated with an incomplete seal. Designed opening gap length of the seal ring is $30-40 \mathrm{~mm}$ in the free state. Measured opening gap length of the used upper seal ring was $24 \mathrm{~mm}$ and the lower seal ring was $16 \mathrm{~mm}$. Consequently, the seal rings could not form a complete seal, causing leakage.

This study was commissioned to identify factors causing the seal ring failure. The seal ring failure was characterized using a series of experiments conducted at both macroscopic and microscopic scales. The same tests and characterization conducted with the new ring helped to analyze the failure mechanisms. 


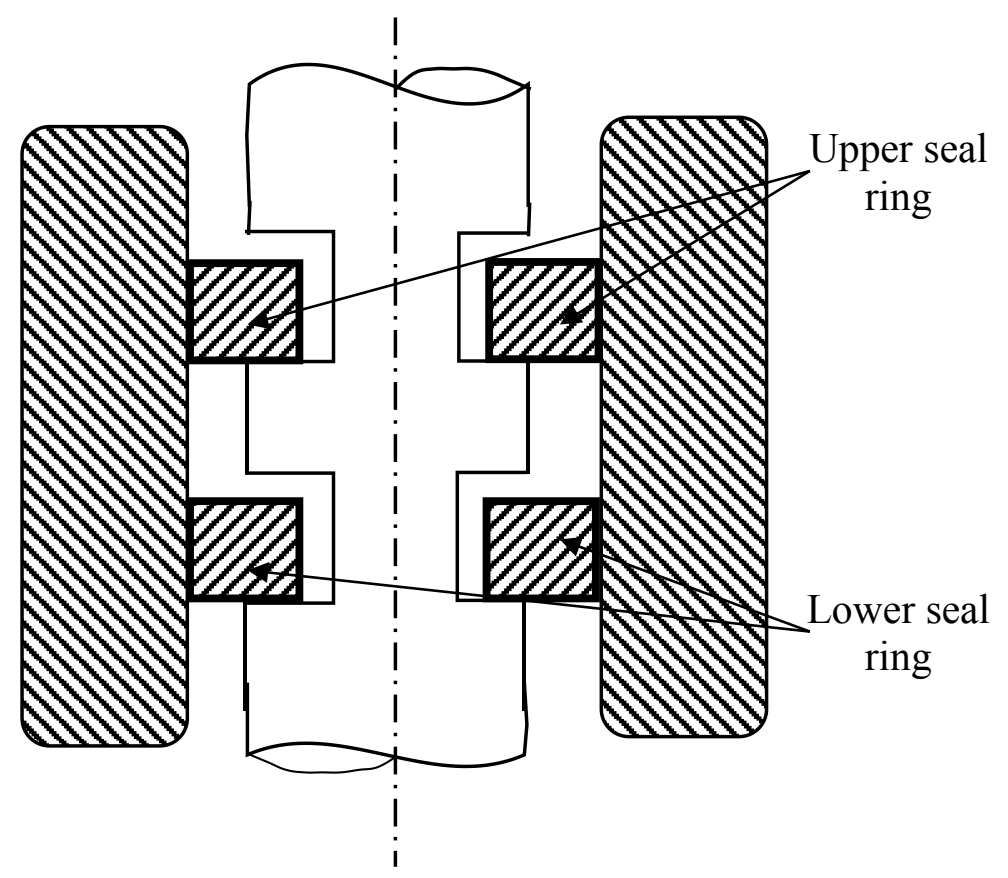

Figure 1. Schematics of the seal rings in the nuclear power turbine system.

\subsection{Steam Turbine}

Steam turbines are used in modern industries. Their reliability and versatility are impressive and meet every demand of most industry fields, along with the steam turbine's constant improvements. The basic principle operation of a steam turbine is a simple converting process. The high-temperature and high-pressure of steam are transformed into effective work. The structures of nozzles in turbine could directly guide the expanding steam flow into high-speed jets while exhausting pressure. These well-formed jets push the movement of rows of blades installed on the rotor. Thus, the blades acting as medium convert the kinetic energy of the expanding steam into the rotation energy of the shaft.

There are two main types of turbines. One is the reaction turbine and another is the impulse turbine, as shown in Figure 2. The steam swells during the blades in both stationary and movement states. Under the effect of steam jet energy of the stationary blades, the moving blades can be regarded as nozzles. A reaction force generated by the pressure drop on it complements 
the steam jet force of the stationary blades during the nozzles movement. These combined forces become the origin of rotation. The reaction turbine has to guarantee the minimum leakage around the moving blades in order to operate efficiently. This demand can be done by making most internal clearances relatively small. Moreover, a balance piston is needed to be installed in the reaction turbine due to the large propulsive force formation. Although the reaction turbine exhibits higher initial efficiency, the reaction turbine is seldom applied in the United States. In contrast, the reaction turbine is widely used in Europe and the rest of the World.

The principle of impulse turbine is different from the reaction turbine. There is little or no pressure drop across its moving blades. The energy of steam is completely transferred to the rotor by the steam jets striking the moving blades. Due to its lack of pressure drop across the moving blades without reaction, large internal clearances can be designed, along with no balance piston. These features make the impulse turbine a rugged and durable machine that can withstand the heavy-duty service of today's mechanical drive applications [3].

(a) Reaction turbine

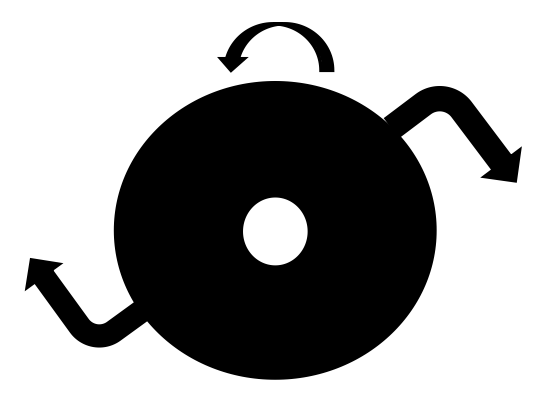

(b) Impulse turbine

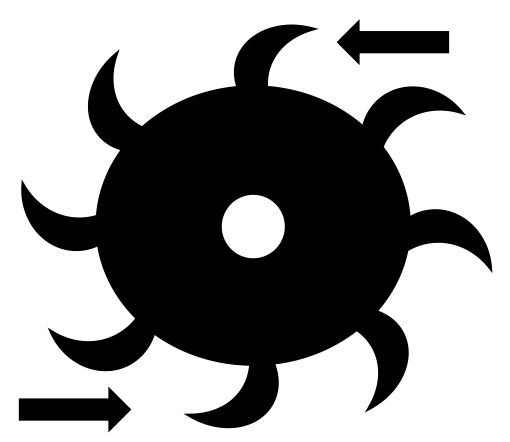

Figure 2. Schematics of two types of turbines: (a) Reaction turbine; (b) Impulse turbine.

There is no doubt that electric energy is the foundation of modern industry. Steam turbines play a critical role in power plants. At present, there is demand for new energy power generation in our society. Nuclear power is a typical representative. However, nuclear power plants are 
facing the challenge of technology and operation safety. For example, it is still an important issue that improving the economic benefits of nuclear steam turbine are generated, meanwhile the cost of generate electricity is reduced [4].

\subsubsection{Steam Turbine Basic Components}

In the system of steam turbines, the delivery power is between 40 and 60 MW. Single-cylinder machines, like condensing turbines, back-pressure turbines and mixed-pressure turbines are used. Other larger steam turbines need more cylinders to extract the steam's energy. There are some basic mechanical components contributing to the operation of this steam turbine.

First, nozzles are an important factor so that the efficiency of a turbine increase. They are made of $12 \%$ or $18 \%$ chrome and $8 \%$ nickel stainless steel and have excellent mechanical strength and corrosion resistance. The diaphragm, together with the nozzle, is split the center line of horizontal and spring-backed labyrinth packing in order to minimize steam leakage.

Second, blades are made of $12 \%$ or $17 \%$ chrome and $4 \%$ nickel stainless steel and have excellent mechanical strength and damping properties. The design theory of blades is based on the combination of aerodynamics and authentic data from diverse tests. The blades usually undergo a large centrifugal force and an excitation force.

Third, compound turbines contain high-pressure and low-pressure cylinders. The low-pressure cylinder in most cases controls a large volume of low-pressure steam through double-flow. Double-flow type can improve the steam flow because of the design dimension of blades limitation. When the cylinders are installed on one shaft, the arrangement is termed a tandem compound. In contrast, cylinders on more than one shaft, are called cross compound.

Finally, the rotor needs to be given high attention in the design, materials and workmanship. Furthermore, the turbine casing is split at the horizontal center line. Casings are made of cast iron 
or steel plate because its different operating steam conditions. The purpose of symmetrical design and simple construction is to reduce the thermal stress created because of temperature changes, along with the casing that would expand and contract concentrically with the rotor $[5-6]$.

\subsection{The Principle of Seal}

Before learning the basic principle of seal in steam turbine, the operation of piston needs to be introduced, shown in Figure 3. The role of the piston is to constitute the combustion chamber with a cylinder head and a cylinder wall while withstanding gas pressure, ultimately driving the crankshaft rotation by piston pin and connecting rod. The piston is usually made of aluminum alloy or gray cast iron, along with light quality and good thermal conductivity. Moreover, the piston needs to exhibit adequate stiffness and strength with reasonable shape and wall thickness. It is better to have a small generating surface and coefficient of thermal expansion, at the same time, possessing a good calorific intensity [7].

It is noteworthy that there are some lantern ring grooves under the piston top. They have three main functions. First, the piston ring can be installed into grooves and cylinder can be sealed with piston top. Second, the lantern ring grooves would prevent the leakage of mixture of combustible gas into the crankcase. Third, the piston ring installed as medium can transfer the heat absorbed at the top of the cylinder wall. The skirt section acts as a guide during the reciprocating motion of the cylinder and bears lateral pressure. 


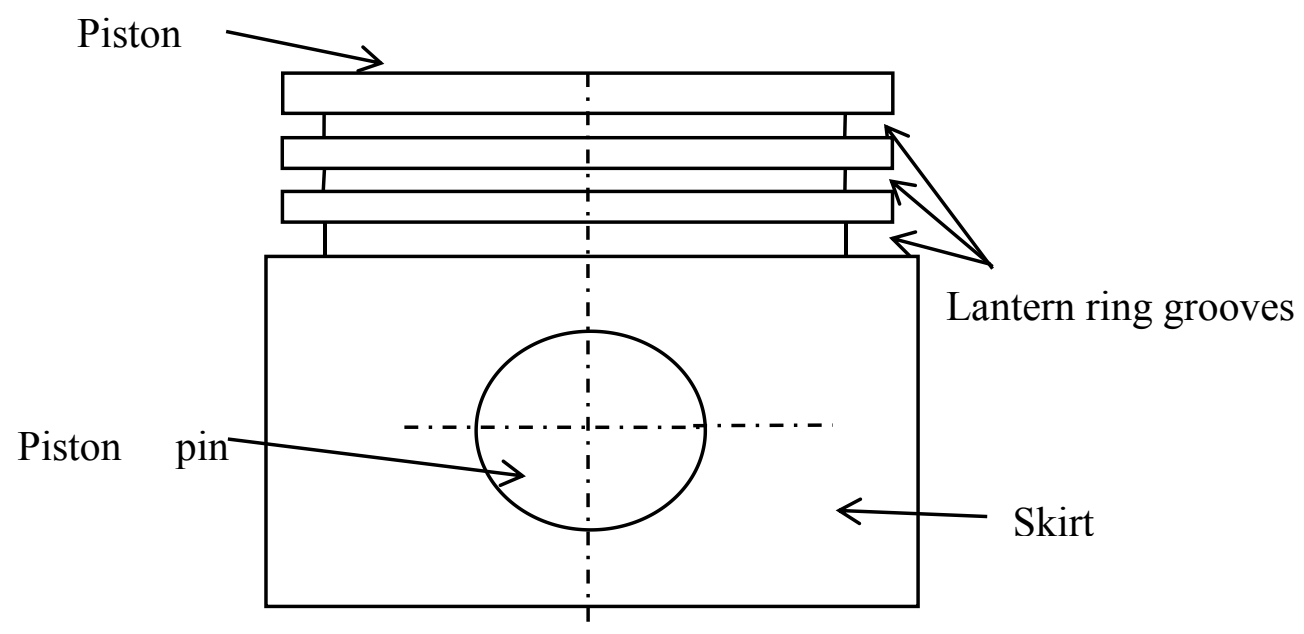

Figure 3. Schematics of piston.

There are two types of piston ring, including gaseous ring and oil ring. The effect of gaseous ring is heat transfer and seal. The operation condition of piston ring is extremely tough, such as high temperature, high pressure and high speed. In the meantime, lubrication condition is rugged. As a result, the service life of piston ring is shorter than any other components. Thus the piston ring must exhibit good elasticity, high strength, good wear and heat resistance. The gaseous ring has a notch, with elasticity, and its external diameter is larger than the diameter of cylinder in the free state. The outer surface of piston ring is glued to the cylinder wall after installing into piston, resulting in forming the first seal surface. After this, high pressure gas in the cylinder directly enters into the side clearance and back clearance. The gas above the side of piston ring presses the piston ring on the lower surface of lantern ring groove, leading to form the second seal surface. Furthermore, the gas strengthened the first seal again, improving the piston ring clinged wall of the cylinder. While there are two seal surfaces formed, the only leakage channel is the notch of piston ring [8]. 
Yet, the gap of the notch is very small, with a staggered certain position, resulting in forming a labyrinth seal gas line. It plays an important role in gas throttles. The schematics of the principal seal is shown in Figure 4.

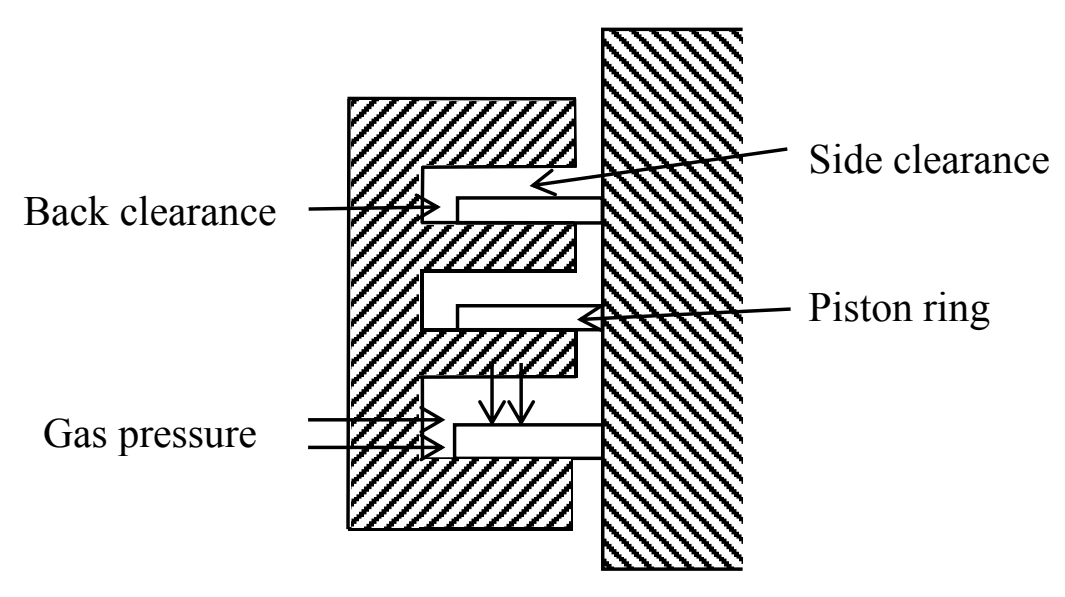

Figure 4. Schematics of principal of seal.

The work environment of the first piston ring is tougher than last piston rings. Thus, the piston ring must have good wear resistance, heat resistance, high strength and impact ductility. In addition, the gap size of notch in piston ring has harsh requirement on dimensions. If the gap size is too large, the effect of the seal will be reduced. Meanwhile, if the gap size is too small, the piston would be locked after thermal dilatation [9]. Overall, the effect of the seal depends on the number of piston rings and ring's material.

\subsection{Deformation}

Metal materials would suffer from external forces during the preparation and work, resulting in the deformation of materials. Additionally, the internal structure and related performance would change with the process of deformation. For example, the stress-strain curve of steel can comprehensively be explained during tensile test, as shown in Figure 5. If the external forces is smaller than elastic limit $\sigma_{\mathrm{e}}$, the materials just would have elastic deformation. 
When the forces is larger than $\sigma_{e}$, but smaller than the tensile strength limit $\sigma_{b}$, the material would plasticaly deform. Finally the material would rupture as the force exceed tensile strength limit $\sigma_{b}$.

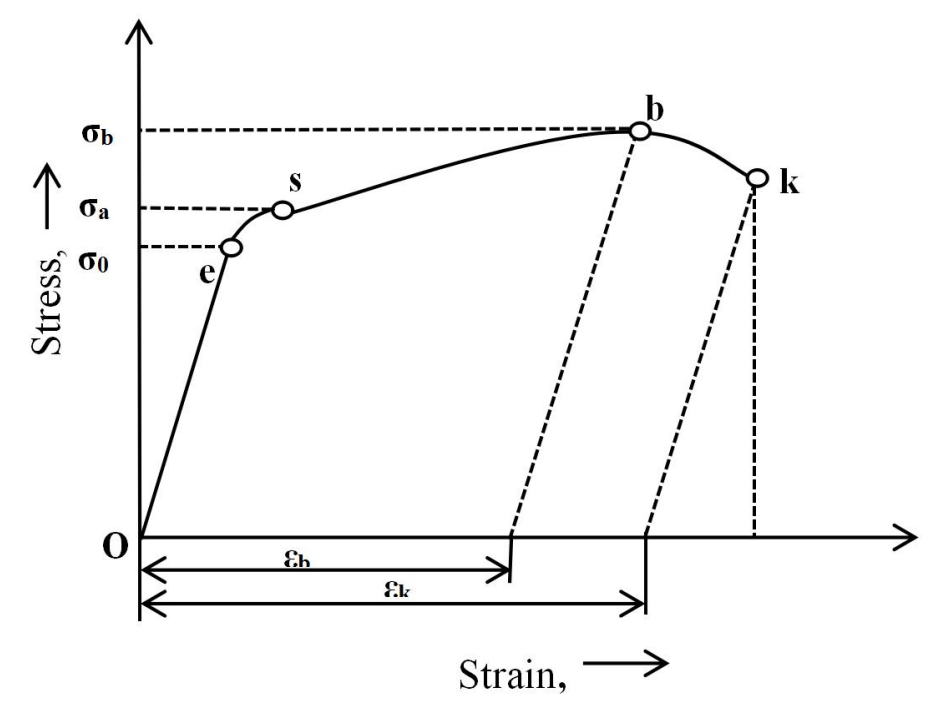

Figure 5. Stress-strain curve of steel during tensile test.

Elastic deformation indicates that the metal could recover original shape after eliminating external forces. The innate character of elastic deformation is that materials' internal atoms deviate from their equilibrium positions under external forces, leading to deformation at the macroscopic scale. However, these internal atoms are able to recover equilibrium positions without external forces. The Hooke law can describe this process as

$$
\begin{gathered}
\sigma=E \bullet \varepsilon \\
\tau=G \bullet \gamma
\end{gathered}
$$

where the formula (1) and (2) are under positive stress and shear stress, respectively. $\sigma$ is the normal stress; $\varepsilon$ is the positive strain; $E$ is the positive elastic modulus; $\tau$ is the shear stress; $\gamma$ is the shear strain; $\mathrm{G}$ is the shear elastic modulus. 
Elastic modulus could represent metallic materials' resistance to elastic deformation. The modulus depends on binding force between atoms. In this project, the new high nickel alloy seal ring possesses good elasticity in order to achieve the seal.

The biggest difference between elastic deformation and plastic deformation is whether materials can recover the original shape after withdrawing forces. Indeed, plastic deformation is an irreversible deformation. The part of deformation that is caused by exceeding elastic limit can not be recovered, resulting in failure [10]. Thus it is necessary to explore the effect of plastic deformation for the material.

Plastic deformation in metals happens by dislocations movement. The internal organization of metal materials would obviously change after plastic deformation. The shape of grains accomodates the change in external shape. For example, equiaxed grains in metals gradually extend along the direction of deformation during rolling down. Grains are altered from polygon to flat parallel or strip. When grains boundaries like fiber, this structure is called fiber structure.

\subsubsection{The Effect of Plastic Deformation on Organization of Metal}

Plastic deformation would impact the microstructure of metal. Dislocations distribution is homogeneous at low plastic deformation or no plastic deformation in metal. However, the situation would be different because of large deformation. The movement of dislocations and their interactions lead to inhomogeneous dislocation distribution. Simultaneously, grains are broken into sub-grains with slightly different orientation. Lots of dislocations gather at the boundaries of sub-grains with less dislocations inside the grains. During the process of plastic deformation, the slip surface destroys grain orientation randomness in polycrystals. This phenomenon is called preferred orientation of grains and this state organization in deformation metal is deformation texture, such as fiber texture and sheet texture, shown in Figure 6. 
Additionally, external forces acting on the metal cause thermal energy during plastic deformation. This function is due to inhomogeneous deformation and lattice distortion. This is called residual stress, exhibiting favorable and unfavorable aspects. Residual stress in most of situations is not accepted in metals, because it would reduce the corrosion resistance of metal and even cause component deformation or rupture [12]. However, after the gear surface suffers from shot blasting, emerged residual compressive stress could improve fatigue strength.

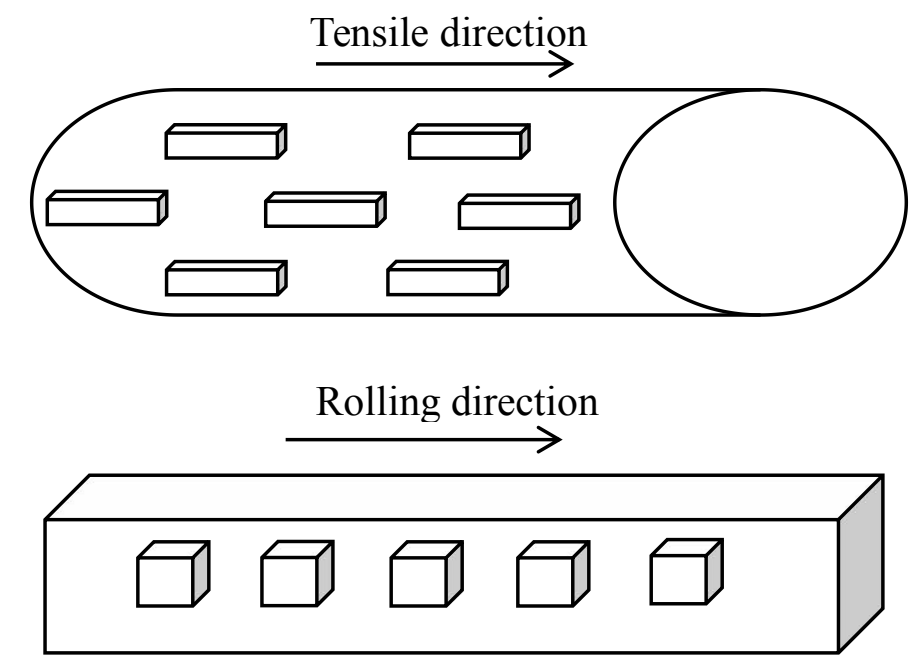

Figure 6. Schematics of fiber texture and sheet texture.

\subsubsection{The Effect of Plastic Deformation on Properties of Metal}

Diversification in the metal internal organization will lead to change of mechanical properties of metal. The hardness and strength of metal are improved with deformation extent increasing, but reduced due to plasticity and toughness. This phenomenon is called work hardening. The cause of work hardening is about density of dislocation and other lattice defects appearing. Density of dislocations constantly raises and the reinforced effect of interaction in dislocation cause resistance of movement increase, ultimately resulting in enhancing the resistance to plastic deformation [13]. The other reason is because grain fragmentation generates lots of subboundary so that dislocation movement is impeded. 
Thus, the strength of metal is enhanced. Work hardening can be applied to improve the strength of metals that are unable to utilize heat treatment, such as $\mathrm{Al}, \mathrm{Cu}$ and some stainless steels.

Plastic deformation also impacts on the physical and chemical properties of metal besides mechanical properties. For example, the specific resistance of metal is increased. Thermal expansion coefficient and thermal conductivity decrease. Moreover, plastic deformation enhances the internal energy of metal.

As described above in the residual stress, the last energy besides causing lattice defects would get stored in the metal. The metal's free energy will be increased after plastic deformation due to this stored energy. Therefore, metal is in a thermodynamically unstable state or metastable state. These metals have ability to recover spontaneously to the steady state, especially with temperature increase. The stored energy as the driving force contributes to this process [14]. In the meantime, the metal might experience three stages, including recovery recrystallization and grain growth with temperature increase, like in Figure 7.

Recovery is a process of generating some sub structure, reflecting in properties and microstructure change in metal when it is heated to low temperature. The mechanism of recovery is about movement of point defects and dislocations upon heating. During low temperature recovery process, vacancies point defects are easier to move, resulting in decreasing the density of point defects. 


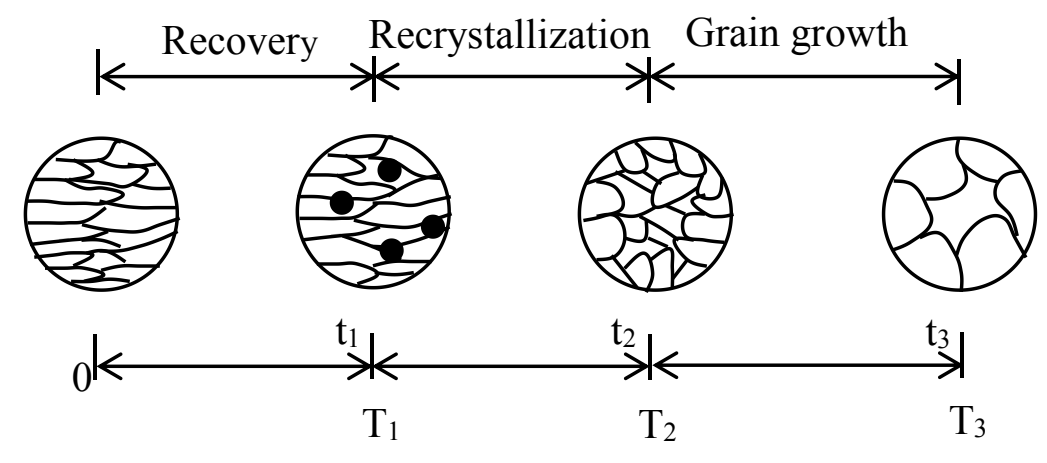

Figure 7. Three stages of organizational change in heating.

The movement of dislocations is a main pattern at high temperature recovery process and produces polygonization. The substance of polygonization is a process where high energy dislocations are reordered to regular array in low energy, as shown in Figure 8.

(a)

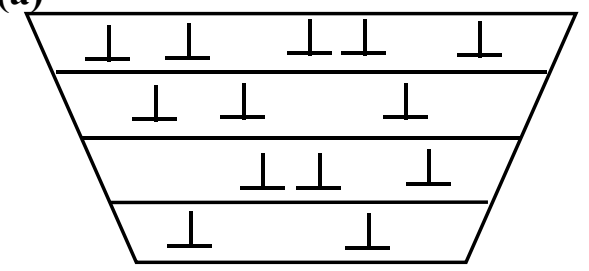

(b)

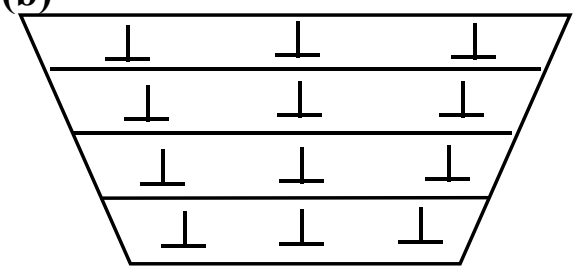

Figure 8. Schematics of dislocation arrangement: (a) Before polygonization; (b) After polygonization.

The recovery process would have an effect on the mechanical properties of metal. The strength and hardness of material slightly decrease and plasticity has a little advance at low temperature [15-16]. Indeed, the principle of stress annealing in actual production is recovery processing to avoid deformation and cracks.

When heating temperature exceed the temperature of recovery, the substrate of deformation organization would generate new and distortionless crystal nucleus, and rapidly form equiaxed grains, ultimately replacing all the deformed texture and returning to the state of complete 
softening. This process is defined as recrystallization. Recrystallization often occurs in high energy area and preferential nucleation, such as grain boundaries, surround the inclusions. There are two patterns of recrystallization nucleation. One pattern called sub crystal nucleation with core derived from sub grain boundaries, occurs at large deformation. Another is convex core. Some parts of the original grain boundaries suddenly grow rapidly and become the core. Convex core often appears in metal with small deformation extent.

One of the important factors in recrystallization is recrystallization temperature. The process of recrystallization is not a thermostatic process, happens at a range of temperatures. Recrystallization temperature is defined as the lowest temperature when completing of recrystallization in a specified period of time after generous deformation (deformation extent $>$ 70\%) [17]. Recrystallization temperature is not a physic at constant, but a temperature range starts from one temperature. The relation between recrystallization temperature and melting point can be described as:

$$
\mathrm{T}_{\mathrm{R}}=(0.35 \sim 0.4) \mathrm{T}_{\mathrm{m}}
$$

Here $T_{R}$ is the recrystallization temperature of metal $(K)$ and $T_{m}$ is the melting point of metal $(K)$.

The strength and hardness of metal would have obviously decrease and increase plasticity of metal in the recrystallization process. Therefore, recrystallization can be used to soften material and for grain refinement.

The final stage is grain growth. This process starts from the end of recrystallization process. With temperature or soaking time increase, the grains will grow by merging with each other. There are two types of grain growth. One is normal growth that would grow uniformly and continuously along with temperature or time increase. 
Another is abnormal growth, which is opposite of normal growth. The process of grain growth is a spontaneous process and depends on grain boundary migration. Finally it is able to make the organization more stable.

\subsection{Common Failure of Materials}

The definition of failure by ASM Handbook has three situations. The first situation is the material can't be repaired completely. Second, the material can't approvingly meet the desired function while in used. Third, the material suffered from serious damage and can't continue to ensure safety and relity during service [18]. Material failure analysis is always a popular topic to avoid the tragic results occurrence, accompanied by an enormous human and economic loss. Moreover, with the rapid development of novel technologies, diverse material failure circumstances need to be overcome. For example, human will never forget the Challenger shuttle disaster and cherish the memory of our excellent astronauts on January 28,1986 . Shortly after liftoff, the space shuttle's external fuel tank collapsed, because the damage of $O$ shape seal ring on the right solid rocket booster, resulting in the combustible gas leaking from the gaps between the skirt and the U shape grooves, burning liquid hydrogen and oxygen fuel of the external tank, ultimately causing the shuttle crash.

Failure analysis is a science that would confirm the interrelation of failure modes, failure defects, failure mechanism and failure cause. Then effective countermeasure would be offered, depending on interrelations, thereby precluding the occurrence of particular types of failure. Furthermore, material failure analysis also leads to the improvement of materials design, manufacturing processes and inspection procedures, so that foundation for exploiting novel material can be achieved. Finally, there are four basic failure modes to be introduced, including fracture, corrosion, wear and deformation. 


\subsubsection{Material Safety Design Criterion and Two Types of Fracture}

Compliance with material safety design criterion can effectively prevent their failure. The first is deformation criterion. The material would have in a certain elastic deformation under the applied load. When the applied load is too large, materials will produce excessive deformation, even warping, resulting in lost of desired function and failure. Thus the deformation of materials must be limited to the elastic deformation range. The formula can be expressed as

$$
\mathrm{F} / \mathrm{EA}=\varepsilon<[\varepsilon]
$$

where $\mathrm{E}$ is elastic modulus, $\mathrm{A}$ is the cross section area, $[\varepsilon]$ is allowable value of elastic strain.

The second is stiffness criterion. This criterion requires that the elastic deformation of the material under applied load does not exceed its allowable deformation. The expression of stiffness criterion is

$$
\left\{\begin{array}{c}
\delta_{\mathrm{i}}<\left[\delta_{\mathrm{i}}\right] \\
\varphi_{\mathrm{i}}<\left[\varphi_{\mathrm{i}}\right] \\
\theta_{\mathrm{i}}<\left[\theta_{\mathrm{i}}\right]
\end{array}\right.
$$

where $\mathrm{i}=\mathrm{x}, \mathrm{y}, \mathrm{z} ; \delta_{\mathrm{i}}, \varphi_{\mathrm{i}}, \theta_{\mathrm{i}}$ are the materials' deflection, deflection angle and torsion angle, respectively. $\left[\delta_{\mathrm{i}}\right],\left[\varphi_{\mathrm{i}}\right],\left[\theta_{\mathrm{i}}\right]$ are the allowable deflection, allowable deflection angle and allowable torsion angle by dividing respective safety coefficients.

The third is strength criterion. When the applied stress is less than the allowable stress, the material is safe, otherwise it's insecure. The expression of strength criterion is:

$$
\left\{\begin{array}{l}
\sigma<[\sigma] \\
{[\sigma]=\sigma_{s} / n_{s}} \\
\sigma_{b} / n_{b}
\end{array}\right.
$$


Here, the value of $[\sigma]$ is the least from both results. $n_{s}$ and $n_{b}$ are the safety coefficients of yield strength and tensile strength, respectively, during the design process.

However, material safety design criterion could not completely eliminate the occurrence of failure. Indeed, many metal materials usually are used in the alternating load. Therefore, these products are subjected to fatigue fracture at low stress, which is lower than the yield stress due to the brittle fracture under fatigue loading. The fatigue fracture is significantly different from static fracture, because it will not produce obvious plastic deformation [19]. In order to reduce the chances of fatigue fracture, the concept of fatigue limit is raised. The fatigue limit $\left(\sigma_{r}\right)$ is the maximum stress that doesn't cause fracture when materials are under the alternating load with the specified number of cycles. Tensile experiment or bending test usually are used to measurement fatigue limit, then drawn the diagram of $\sigma-\mathrm{N}$ curve in Figure 9. Additionally, the reasonable selection of materials is needed, to avoid using brittle materials, and the suitable structures could reduce stress concentration.

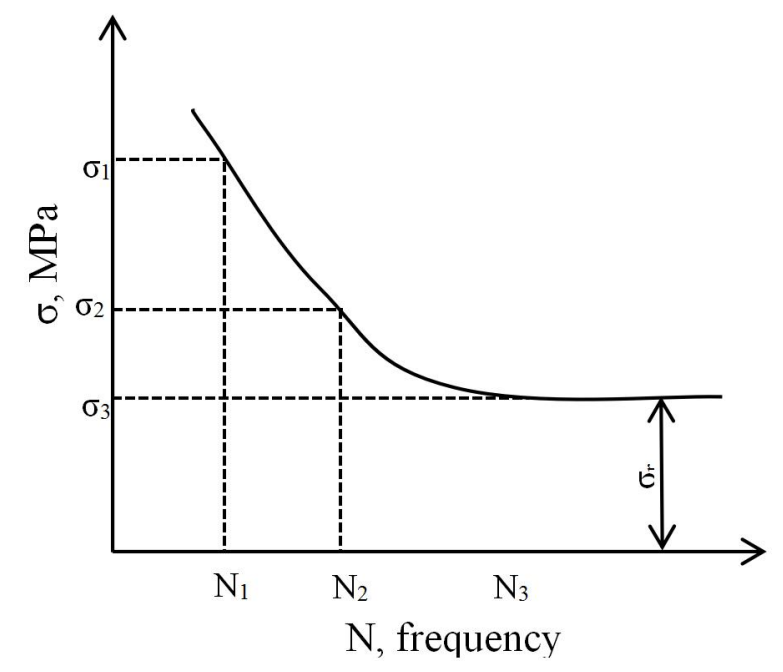

Figure 9. Curve of fatigue. 
Creep is another failure besides fatigue fracture. The creep is when materials undergo slow plastic deformation because of constant temperature and stress in the long-term. The creep process can be described by the creep curve, as in Figure 10. The point a is the instantaneous strain after applying stress to the specimen, without creep. The strain that develops over time from the point a is creep, and the slope of any point on the curve represents the creep rate at that point. The first stage from a to $\mathrm{b}$ is called decelerating phase or unstable phase where the rate of creep is gradually reduced with time. The second stage from $\mathrm{b}$ to $\mathrm{c}$ is a constant velocity phase or steady stage with invariant creep rate. The creep rate would be accelerated until fracture from point $\mathrm{c}$ to point $\mathrm{d}$, called accelerate stage or final stage.

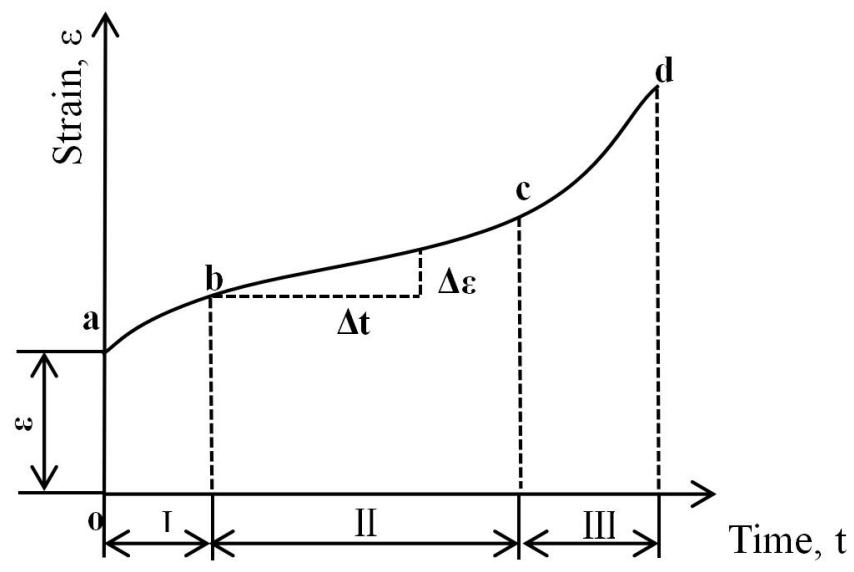

Figure 10. Curve of creep process.

Exactly as the fatigue limit, creep limit, duration strength and relaxation stability can estimate the creep performance. Creep limit is defined that the value of stress, which causes the total deformation in a specified temperature and time conditions. The duration strength is the maximum carrying capacity, which can be maintained without failure in the specified temperature and time conditions. Temperature is an important factor for creep. Selecting reasonable materials with good thermal stability can prevent creep when working in the high temperature, simultaneously avoiding the fluctuation of temperature [20-21]. 
Fracture induced material failure is harmful, accompanied by catastrophic consequences. Therefore, when the premonition of fracture is inspected, effective methods must be present immediately in order to reduce the occurrence probability of failure.

\subsubsection{Corrosion}

The detriment of corrosion could involve every industry. There are various types of corrosion problems where the metal is used. Corrosion deterioration is caused by the chemical reaction or electrochemical reaction between materials and the environment. Corrosion can be divided into chemical reaction and electrochemical reaction, depending on the corrosion mechanism. When there is no electron transfer during the whole reaction and the valence unchanged, the process is called chemical reaction. In another words, the process of reaction does not produce current. The electrochemical reaction is the exact opposite to chemical reaction. At the same time, current is produced in the electrochemical reaction.

The types of corrosion damage contain uniform and local corrosion. The uniform type is a common type, such as metal in the atmosphere and the oxidation reaction in high-pressure steam pipe. The corrosive medium would uniformly reach the surface of the metal and react by electrochemical method. Generally, the uniform type would impact the whole surface, along with slow and uniform corrosive rate. Further to say, the harmfulness of uniform type is light. As a result, the uniform corrosion resistance can be improved through the surface coating, corrosion inhibitor, cathodic protection, and selecting the appropriate material [22-23].

On the contrary, the local type corrosion is a great hazard and difficult to prevent, because it occurs in the micro-area of material with hidden property and large corrosion rate. The local type, such as pitting, intergranular and stress corrosion cracking, can be distinguished by different destruction forms. 
The formation mechanism of pitting corrosion is about $\mathrm{Cl}^{-}, \mathrm{F}^{-}$ions and other halogen ions. Indeed, the pitting corrosion is an autocatalytic process. The metal in the small hole is dissolved so that the concentration of $\mathrm{H}^{+}$ion in the hole is increased. Although redox reaction does not occur, the adjacent surfaces of the hole generate oxygen's cathode reduction reaction, resulting in the pores, rapid extent along the depth direction. The process can be described as:

$$
\begin{array}{cl}
\mathrm{M}^{+}+\mathrm{Cl}^{-} \rightarrow \mathrm{MCl} & \text { (inside of pitting) } \\
\mathrm{MCl}+\mathrm{H}_{2} \mathrm{O} \rightarrow \mathrm{MOH}+\mathrm{H}^{+}+\mathrm{Cl}^{-} & \text {(outside of pitting) }
\end{array}
$$

Therefore, the measure of prevention pitting corrosion can select corrosion resistant alloy material and adopt amendatory medium with reducing the concentration of $\mathrm{Cl}^{-}$. Moreover, passivation material surface is another method to strengthen the pitting corrosion resistance.

The intergranular corrosion is the corrosive behavior along the grain boundaries or grain boundaries when the metal is in certain corrosive media, resulting in lower strength of intergranular bonding. There are impurity elements at grain boundaries. Thus, the concentration of the certain alloying element will be reduced because the enrichment or precipitation of a certain phase, like $\mathrm{Cr}$, causing the chemical stability of lack alloying elements decline around the grain boundaries or adjacent narrow areas [24]. In the meantime, the media is more aggressive in these areas, resulting in intergranular corrosion. After the occurrence of intergranular corrosion, the dimensions of metal are almost unchanged, while the metal's strength and ductility significantly decrease, sometimes with the metal loss.

As a result, the common prevention intergranular corrosion methods have alloying method, reducing the content of carbon and heat treatment. The alloying is that adding elements, which can form strong carbon compounds, such as $\mathrm{Ti}$ and $\mathrm{Nb}$. Solution treatment can also improve the resistance of intergranular corrosion. 
The stress corrosion indicates that metal can experience brittle fracture by the joint action of tensile stress and corrosive medium. The corrosive behavior of some materials is minuscule in medium without tensile stress. However, even ductile metal can experience brittle rupture when the tensile stress reach a certain level, causing disastrous consequences without any premonition [25]. There are three conditions that facilitate stress corrosion cracks, including tensile stress, sensitive material, and specified medium. The origin of cracks generally derives from the bottom of pitting corrosion.

Therefore, the value of external stress need to be control led along with the critical cracking stress. Non sensitive material could replace the material for sensitive stress corrosion. In addition, electrochemical protection could also avoid stress corrosion.

\subsubsection{Wear}

The meaning of wear is that surface dimensions of components change with material loss during friction process. Wear is a complex phenomenon, involving surface physics, solid mechanics, lubrication, capillary chemistry, materials science and metallurgy. Meanwhile, wear is affected by many factors. Internal factors contain heat conductivity, surface energy and hardness, and microstructure. External factors consist of pressure, temperature, chemical environment, slip velocity and surface topography. In the practical working, wear can be divided into abrasive, adhesive, erosive, fretting, fatigue, corrosive and cavitation wear. In the steam turbine work, adhesive, erosive and fatigue wear often appear [26].

Adhesive wear refers to the two uneven metal surfaces come in contact and rubbing each other during relative motion between metals, causing plastic deformation in uneven surfaces and finally join together with local constant increase high temperature. Ultimately these bonds are broken and produce cavities on one surface, simultaneously depressing on the second surface. 
Abrasive particles detach and rub against the surface, contributing to wear. The adhesive wear mechanism in piston system is shown in Figure 11.

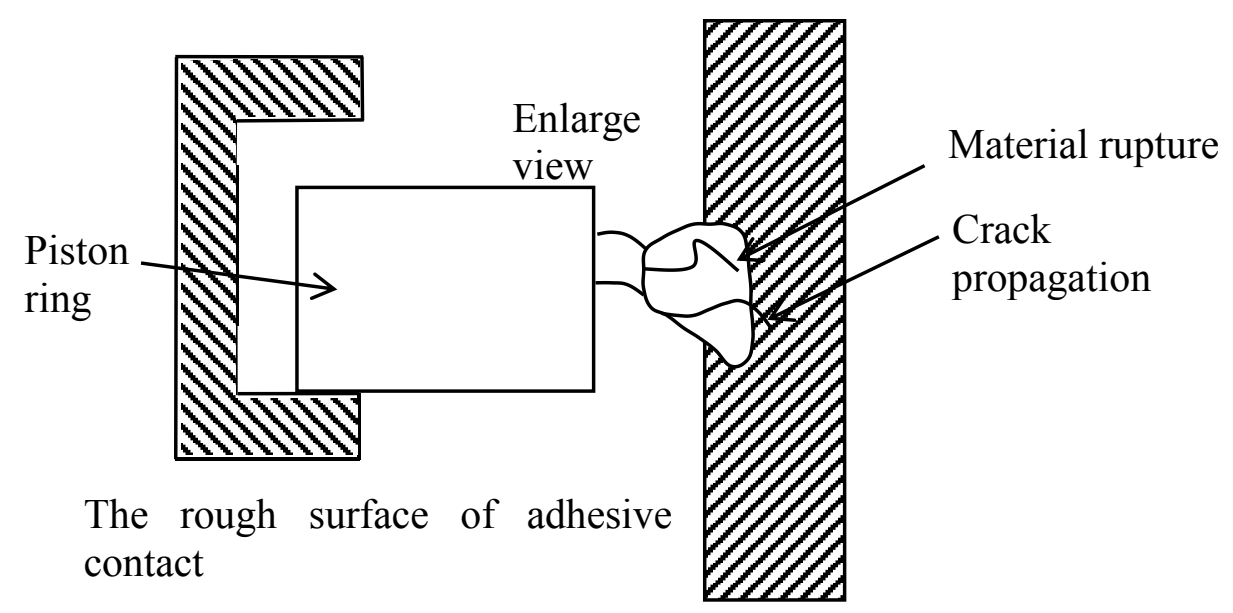

Figure 11. Schematics of adhesive wear between piston ring and cylinder wall.

Generally, adhesive wear possesses high rate of wear and serious destructiveness. As a result, some methods can be adopted to prevent adhesive wear, such as rational selection of material, enhancing the hardness of surface and reducing the roughness and contact pressure.

Erosive wear can be defined as the fluid (liquid or gas) with particulate scours the solid surface, causing the local surface material loss and damage. In order to calculate the mass loss during erosive process, the mass loss rate can be expressed as:

$$
\mathrm{W}_{\mathrm{e}}=\mathrm{kV}^{\mathrm{n}}+\mathrm{W}_{\mathrm{w}}+\mathrm{W}_{\mathrm{c}}+\mathrm{W}_{\mathrm{w}} \times \mathrm{W}_{\mathrm{c}}
$$

Here, $\mathrm{W}_{\mathrm{e}}$ is the total loss rate of the pipe unit surface during erosion $(\mathrm{mg} / \mathrm{g})$; $\mathrm{V}$ is the velocity of particulate $(\mathrm{m} / \mathrm{s}) ; \mathrm{n}$ is the constant of material; $\mathrm{k}$ is a coefficient depending on the size and shape of the particulate, erosion angle, temperature, pressure and other factors.

Fatigue wear can be described when periodic load increases the stress until exceeding the fatigue strength of the material in the contact zone when the friction surfaces undergo relative sliding or rolling against each other. The weak point of surface would produce cracks and 
gradually propagate. The process is similar to the fatigue rupture with cracks initiation and propagation. The preventive measure can enhance the hardness and purity of the material and reduce the roughness of the surface. 


\section{CHAPTER 2: MATERIAL MEASUREMENT METHODS}

\subsection{Measurement at Macroscopic Scale and Specimen Preparation}

The obvious distinction between new seal ring and used seal ring is metallic luster. The used seal ring basically lost its metallic luster because of corrosion. It is essential to measure the basic dimensions at macroscopic scale for comparison. Therefore, macroscopic ring dimensions, such as external and internal diameters, width, opening gap length and gap lateral displacement of the used and the new seal rings, were measured using calipers.

After collecting these dimensions, specimens were cut from the new and used seal rings (Figure 12), grinded with emery paper to 2000 grade and polished in order to observe the microstructure. After grinding and polishing, specimens were etched in mixed acid glycerin solution $\left(\mathrm{HCl}: \mathrm{C}_{3} \mathrm{H}_{8} \mathrm{O}_{3}: \mathrm{HNO}_{3}=3: 3: 1\right.$ ratio) for $20 \mathrm{~s}[27]$.
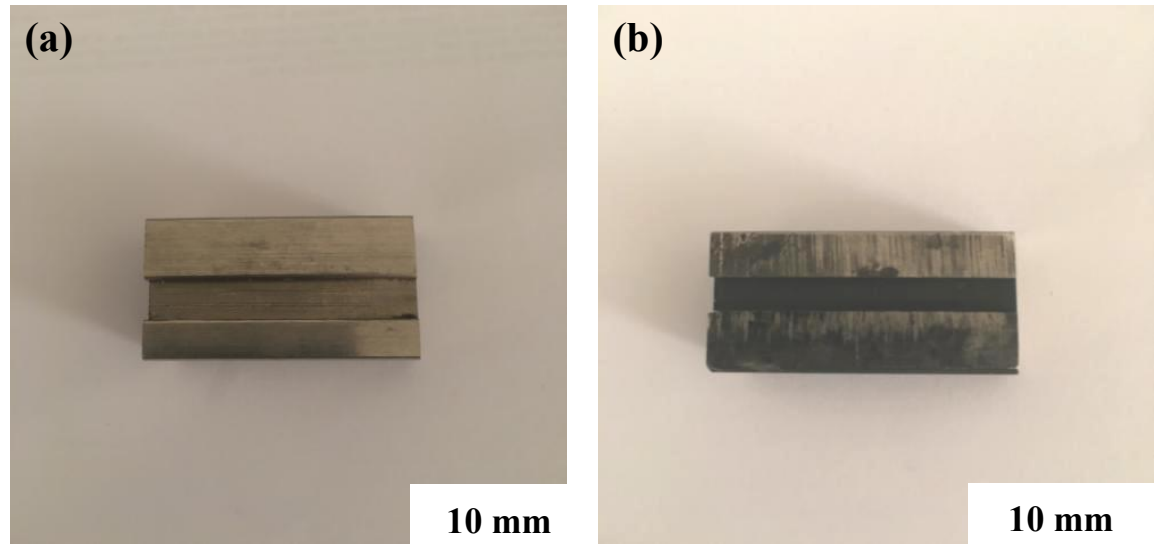

Figure 12. Specimens cut from: (a) New seal ring; (b) Used seal ring. 


\subsection{The Hardness and Elastic Modulus of Seal Ring Test}

The hardness of material is an ability to resist surface deformation. Hardness is an important factor to the seal ring, such as a significant impact on wear. There are three general types of hardness test, including Brinell hardness test, Rockwell hardness test and Vickers hardness test.

The first step of brinell hardness test is to exert a certain load. Then the indenter made by quenching steel ball or sintered carbide ball would be pressed into the surface of the materials and maintain a certain time before unloading. Finally, the hardness can be calculated by the indentation diameter. The formula is:

$$
H B=\frac{P}{A}=\frac{2 P}{\pi D\left(D-\sqrt{D^{2}-d^{2}}\right)}
$$

Here, $\mathrm{P}$ is the load; $\mathrm{A}$ is the area of indentation; $\mathrm{D}$ is the diameter of steel ball; $\mathrm{d}$ is the indentation diameter.

Brinell hardness test is suitable to measure the metallic materials with Brinell hardness less than $450 \mathrm{HB}$, especially for test material with large difference in hardness, such as iron casting.

The Rockwell hardness test is distinguish from Brinell hardness test. Rockwell hardness test utilizes the indentation depth to measure the hardness during indenting the surface. The formula is:

$$
H R=\frac{k-h}{0.002}
$$

where $\mathrm{h}$ is the deep of indentation; $\mathrm{k}$ is the constant that depending on indenter, listed in Table 1. 
Table 1. Three common types of Rockwell hardness values.

\begin{tabular}{|c|c|c|c|c|}
\hline $\begin{array}{c}\text { Rockwell } \\
\text { Hardness }\end{array}$ & Indenter & Full payload, N & K & $\begin{array}{c}\text { Measurin } \\
\text { g range }\end{array}$ \\
\hline HRA & $120^{\circ}$ Diamond cone & 600 & 0.2 & $60-85$ \\
\hline HRB & $\begin{array}{c}\Phi 1.5 \mathrm{~mm} \\
\text { quenching steel ball }\end{array}$ & 1000 & 0.26 & $25-100$ \\
\hline HRC & $120^{\circ}$ Diamond cone & 1500 & 0.2 & $20-67$ \\
\hline
\end{tabular}

Depending on indenter and the value of the load, the Rockwell hardness can be divided into HRA, HRB and HRC. The type of HRA can be used to measure cemented carbide material or surface chilled steel. HRB is fit for measuring nonferrous metals and normalized steels. HRC is propitious to test modulated steel and chilled steel.

Vickers hardness test uses $136^{\circ}$ angle four sided pyramid as indenter to test hardness by measuring the diagonal line of press indenter. The formular is:

$$
H V=\frac{1.8544 P}{d^{2}}
$$

where $\mathrm{P}$ is the load; $\mathrm{d}$ is the value of diagonal line.

The Vickers hardness test could measure soft material and hard material because of high hardness of the indenter. Moreover, it is also able to test sheet metal and surface of material due to small indentation.

\subsubsection{Nanoindentation}

With the development of modern microelectronic materials science, the traditional measurement methods have gradually exposed their limitations. The novel nanoindentation method is a good solution to offset these defects of traditional measurement. Nanoindentation instrument with high load and displacement resolution, has been widely applied to the surface micro-nano-mechanical properties testing of materials. 
It is necessary to know operation principle of nanoindentation before using it to test hardness. A prescribed load is applied to an indenter during contacting the specimen. The depth of penetration is measured when the load is applied. The area of contact at full load is determined by the depth of the impression and the known angle or radius of the indenter [28]. The hardness is found by dividing the load by the area of contact. Shape of the unloading curve provides a measure of elastic modulus.

As a result, after putting the specimen of used and new seal ring on the sample tray, the test was started. The schematic of testing process and curve of load-displacement are shown in Figure 13.

(a) Indenter

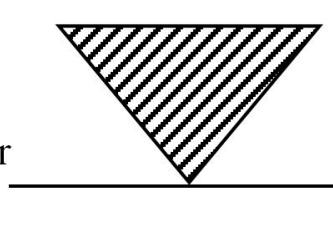

At surface

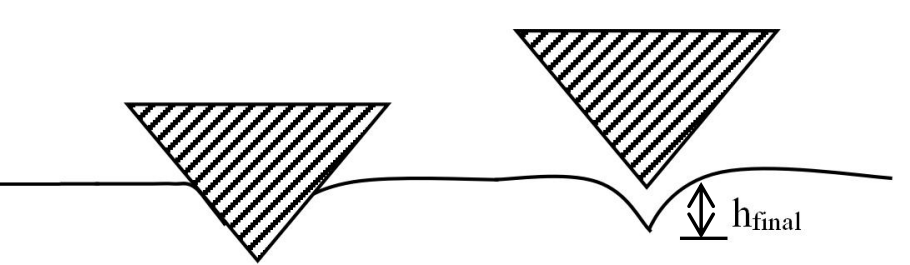

$$
\text { Under load }
$$

$$
\text { After unloading }
$$

(b)

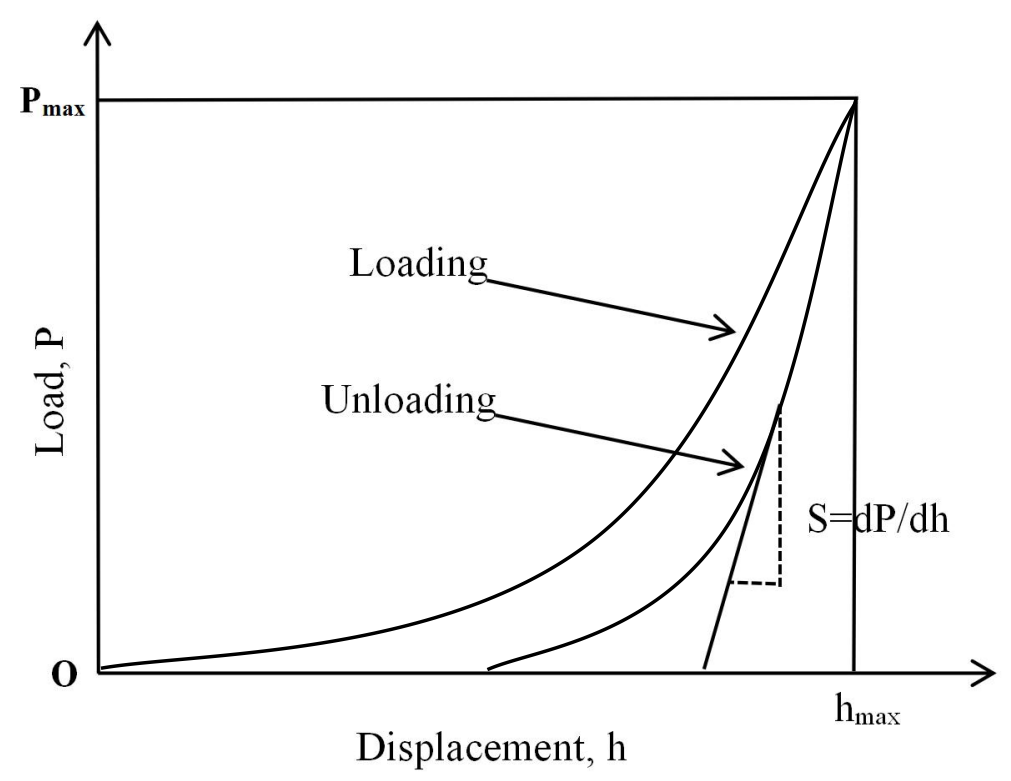

Figure 13. Schematics of nanoindentation technique: (a) Indenter at surface; (b) Curve of load-displacement. 
Computer program controls the continuous variation of load, thus nanoindentation could measure real-time indentation depth. Then utilizing the mathematics formulas as:

$$
\begin{gathered}
H=\frac{P}{A} \\
S=\frac{d F}{d h}=\frac{2}{\sqrt{\pi}} E_{R} \sqrt{A} \\
\frac{1}{E_{r}}=\frac{1-v^{2}}{E}+\frac{1-v_{i}}{E_{i}}
\end{gathered}
$$

Formula (11) is used to calculate the hardness; the formula (12) is to calculate Young's modulus; the elastic modulus of material can be obtained by formula (13).

\subsection{Scanning Electron Microscope and Energy-dispersive Spectrometer}

The metallograph of failed and new seal ring can be obtained by optical microscopy. However, it is essential to inspect the surface micrograph of the specimen. Thus the scanning electron microscope (SEM) plays a vital role in conducting measurements. SEM is a kind of electronic instrument which has been developed rapidly in recent decades. It would examine the microscopic structure by scanning the surface of materials with high resolution and great depth of field [29].

The operation can be described three basic steps. First, a stream of electrons would be formed by electron source and accelerated towards a specimen using a positive electrical potential. Then, the apertures and magnetic lenses would focus the stream onto the specimen. Finally, interactions occur inside the irradiated area of the sample, and images can be collected in a suitable detector. The structure of SEM schematic is shown in Figure 14. 


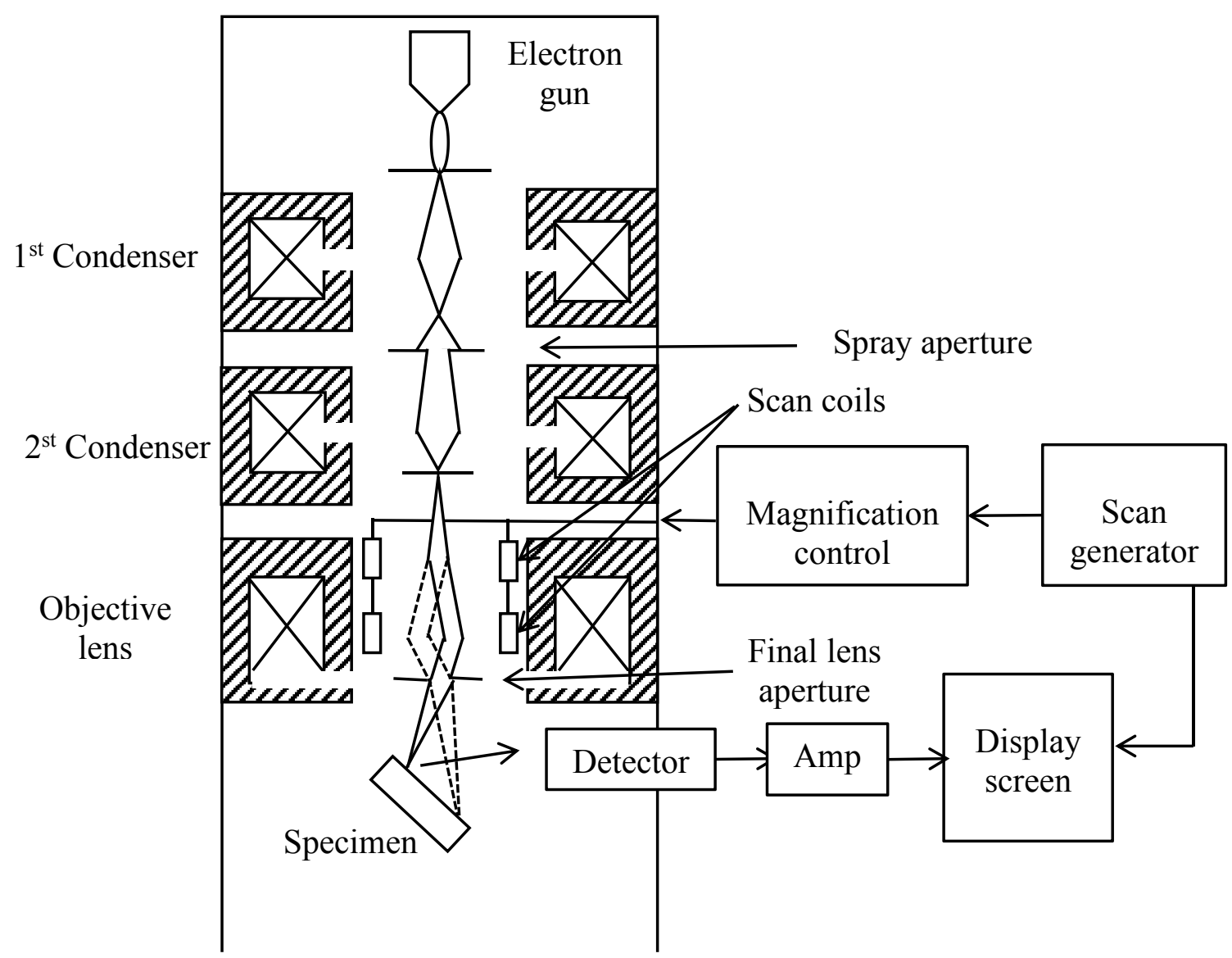

Figure 14. Schematics of SEM structure.

High-speed electrons form the incidence electron probe bombard the surface of specimen, resulting in generating secondary electrons, Auger electrons, characteristic X-rays and continuum X-rays, backscattered electrons, transmitted electrons, and electromagnetic radiation in the visible, ultraviolet, and infrared regions. Therefore, the desired information of the specimen, such crystal structure, morphology and composition, can be obtained due to the interaction between electrons and substance, shown in Figure 15. 


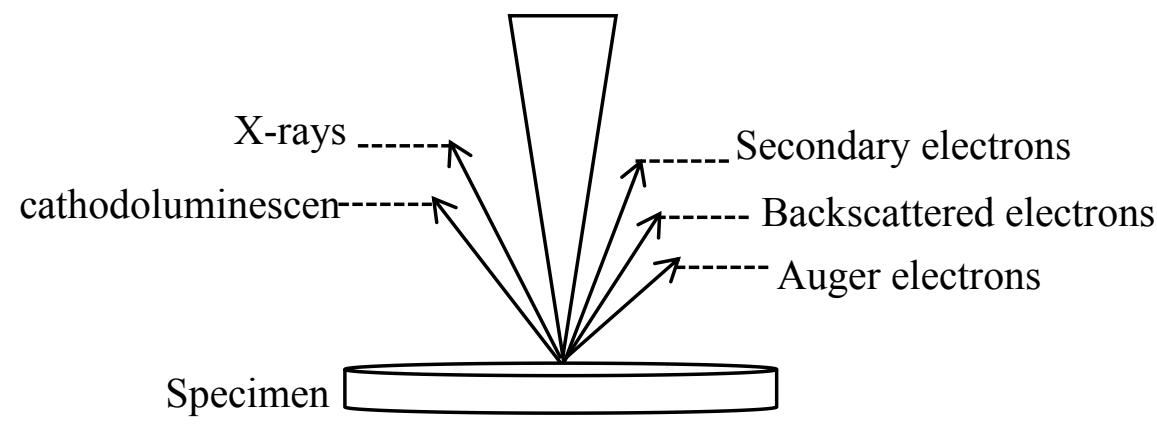

Figure 15. Interaction between electron beam and sample above the surface.

The advantages of using SEM are images that are obtained with large magnification range from $10 \times$ to $1 \mathrm{M} \times$ and large depth of field ( $\mathrm{nm}$ to $\mathrm{cm}$ ). Magnification refers to how large an object can be made.

$$
\text { Magnification }=\text { image size/ object size }
$$

The meaning of resolution is the closest distance between two points that can clearly be resolved as separate entities through the microscope

$$
r=\frac{d_{1}}{2}=\frac{0.61 \lambda}{\mu \sin \alpha}=\frac{0.61 \lambda}{N A}
$$

where $\lambda$ is the wavelength of illuminant; $\alpha$ is semi-angle; $\mu$ is the index of refraction; and NA is the numerical aperture.

The resolution of SEM imaging is determined by the cross-sectional diameter of the scanning probe. Therefore, the probe size impacts on the size of features on the specimen surface to be resolved. To obtain high resolution, probe size needs to be minimum. The probe diameter can be approximately expressed as $d_{p}$

$$
d_{p}=\left(\frac{4 i_{p}}{\beta \pi^{2} \alpha_{f}^{2}}\right)^{1 / 2}
$$


where $i_{p}$ is the probe current; $\beta$ is the beam brightness, which is controlled by the electron source; and $\alpha_{\mathrm{f}}$ is the convergence angle of the probe, which is determined by the final aperture diameter and the distance between aperture and specimen surfaces, called the working distance.

The beam brightness $\beta$ is determined by type of electron gun used. Moreover, the brightness is proportional the acceleration voltage of the gun

$$
\beta \propto e V_{0}
$$

where $\mathrm{e}$ is the charge of an electron; $\mathrm{V}_{\mathrm{o}}$ is the acceleration voltage.

As a result, to obtain a minimal probe size, the beam brightness and convergence angle need to be increased. But the convergence angle is not simply increased because of optical problems. Finally, the minimum probe size is approximated as $d_{\min }$

$$
d_{\min }=K C_{S}^{1 / 4}\left(\frac{i_{p}}{\beta}+\lambda^{2}\right)^{3 / 8}
$$

where $\mathrm{K}$ is a constant close to unity; $\mathrm{C}_{\mathrm{s}}$ is the spherical aberration coefficient; $\lambda$ is the wavelength of the electrons.

Thus, the minimal probe size can be obtained by decreasing wavelength and spherical aberration and increase the brightness of electron illumination.

The depth of field is how much of the object that can be looked at remains in focus at the same time.

$$
D_{f}=\frac{1.22 \lambda}{\mu \sin \alpha \tan \alpha}
$$

where $\lambda$ is the wavelength of illumination.

There are two main factors, the working distance and aperture size affecting the depth of field in Figure 16. 


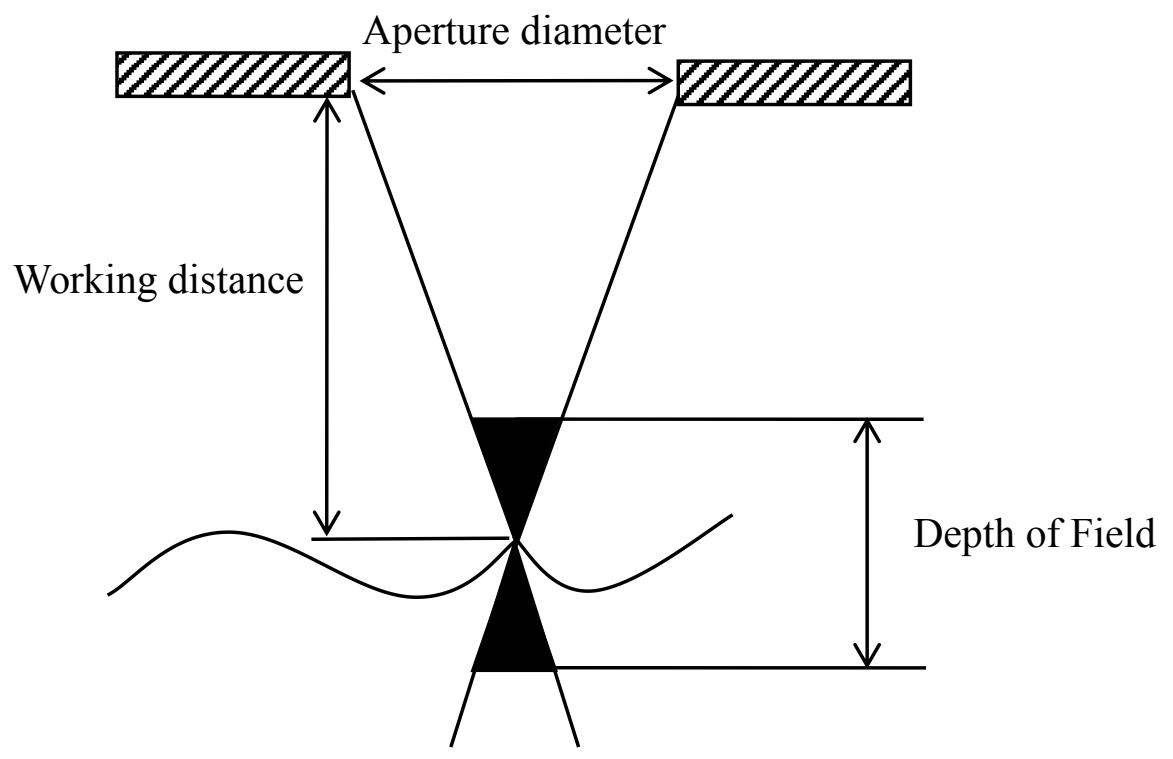

Figure 16. Relationship between depth of field, aperture diameter and working distance.

In the meantime, the depth of field is related to the resolution $\mathrm{R}$ and convergence angle $\alpha$ of the objective aperture.

$$
D_{f}=\frac{2 R}{\tan \alpha}
$$

Indeed, the higher depth of field and higher resolution can not be obtained together. It is wise to select an intermediate aperture size and intermediate working distance, unless a very large depth of field or very high resolution is required [30].

As mentioned above, the information of the sample can be obtained from the characteristic X-rays that are excited from the material. Energy-dispersive spectrometer (EDS) is used to analyze elements of seal ring in this project. The advantages of EDS is fast analysis and low cost. Moreover, an EDS system is simple as shown in Figure 17. 


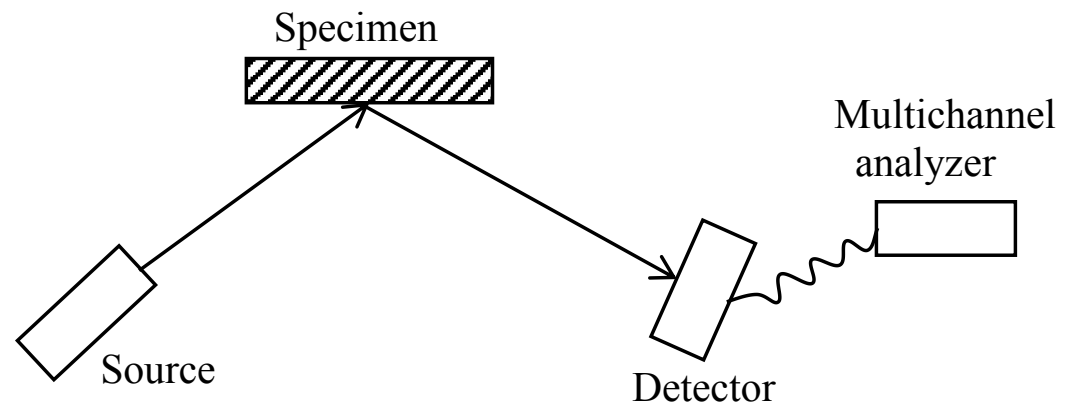

Figure 17. The basic structure of EDS system.

\subsection{In-situ SEM Tensile Test}

The purpose of in-situ SEM tensile test is to observe the whole tensile process under scanning electron microscope. The sample chamber is equipped with tensile table, allowing to research the initiation and propagation of cracks during the tensile test. The sample is generally non-standard samples because of load constraints. In this project, the machine model is Camscan 3400. The maximum load could reach $5000 \mathrm{~N}$, but it usually won't exceed $4000 \mathrm{~N}$. The size of sample need to meet the demand of tensile table, shown in Figure 18.

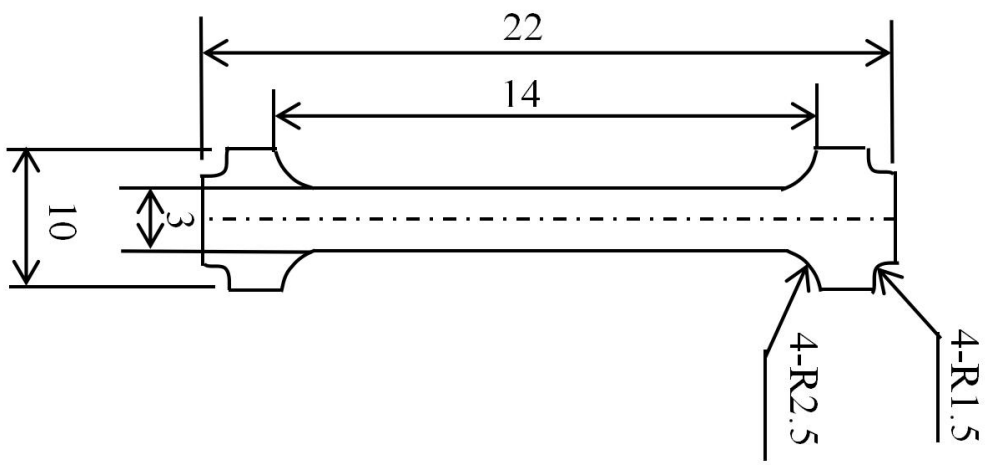

Figure 18. The size of simple for in-situ SEM tensile test. Dimensions are in $\mathrm{mm}$. 
Thus, the failed seal ring and new seal ring are processed depending on the size demand of Camscan 3400. In addition, the middle part of sample could have breach and the thickness of sample is smaller than $1 \mathrm{~mm}$. The observation surface needs to be polished. The loading rate can be controlled and the state of load could be maintained after the cracks are generated. As a result, the microstructure of the crack tip is observed in situ and the relationship between the crack propagation path and the microstructure is recorded. 


\section{CHAPTER 3: EXPERIMENT RESULTS AND DISCUSSION}

\subsection{Macroscopic Dimensions Examination}

Macroscopic and microscopic analyses were used to identify material failure. Table 2 lists measured diameter, width, opening gap length and gap lateral displacement of the failed and the new seal rings, shown in Figure 19. While there are no obvious differences in the rings diameter, the opening gap length of the failed ring is larger, along with the gap lateral displacement.
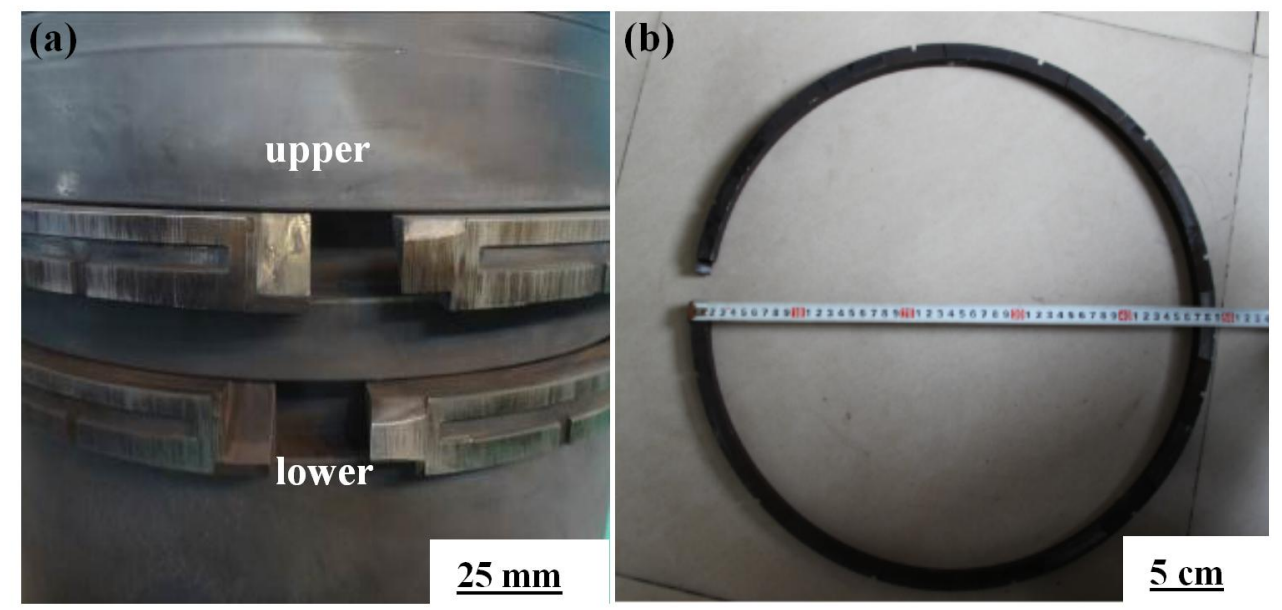

Figure 19. (a) Upper and lower seal rings on the turbine; (b) Macroscopic ring dimensions measurements.

Table 2. Seal ring dimensions.

\begin{tabular}{|c|c|c|c|}
\hline Parameter & Used 1 & Used 2 & New \\
\hline External diameter, cm & 48.9 & 49.3 & 49.52 \\
\hline Internal diameter, cm & 46.1 & 46.39 & 46.25 \\
\hline Opening gap length, mm & 25.94 & 31.86 & 29.68 \\
\hline Width, cm & 1.89 & 1.88 & 2.11 \\
\hline $\begin{array}{c}\text { Gap lateral displacement, } \\
\text { mm }\end{array}$ & 7.38 & 3.04 & 0 \\
\hline \multicolumn{2}{|l}{} & & \\
\hline
\end{tabular}


Thus, it is apparent that the used rings have undergone macroscopic plastic deformation, contributing to seal ring failure. Moreover, the used seal ring might be subject to wear so that its width is smaller than the new seal ring.

\subsection{Relationship Between the Opening Gap Length and the Applied Load}

The opening gap length of the seal ring was measured at different externally applied loads. The top of failed seal ring was fixed first and then external load was applied to the bottom of failed seal ring in order to measure opening gap length by the calipers, shown schematically in Figure 20, and the results are listed in Table 3.

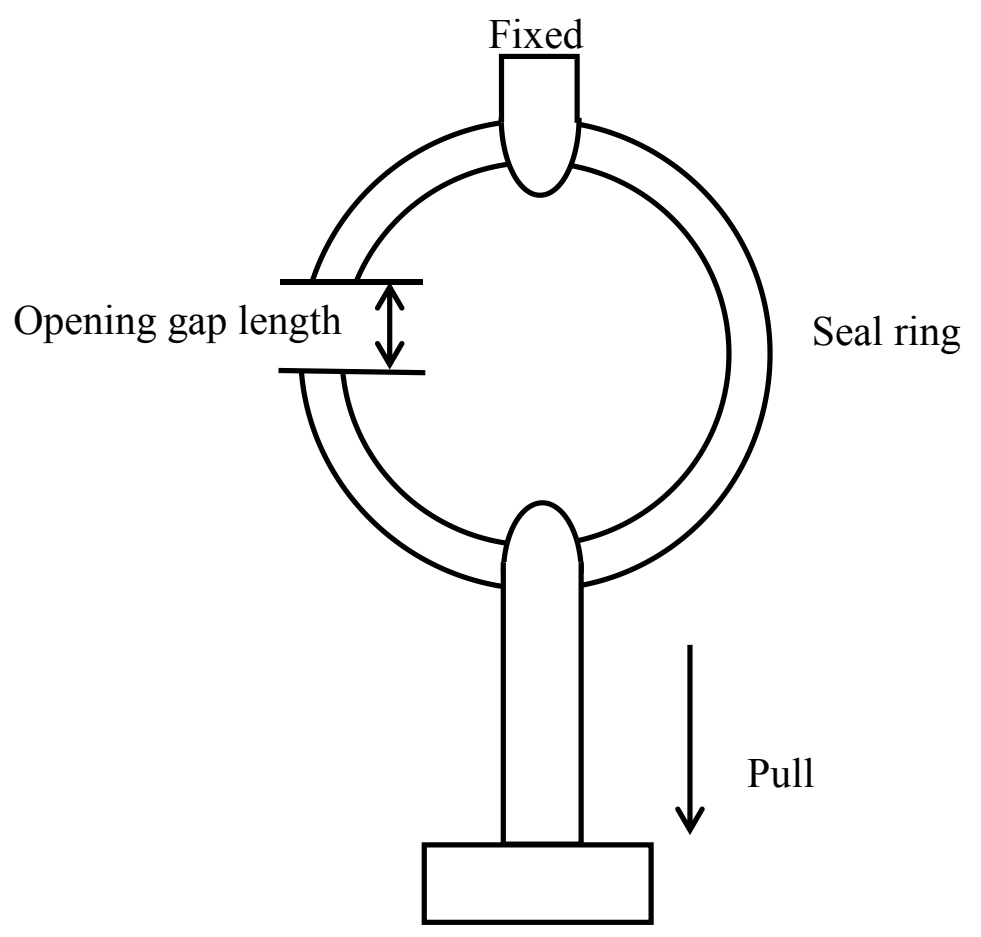

Applied load

Figure 20. Schematics of the opening gap length measurement method. 
Table 3. Relationship between the externally applied load and the seal ring opening gap length.

\begin{tabular}{|c|c|c|c|c|c|c|c|c|c|c|c|}
\hline $\begin{array}{c}\text { Applied load, } \\
\text { kg }\end{array}$ & 0 & 0.6 & 1.6 & 2.6 & 3.6 & 5.6 & 7.6 & 10.6 & 15.6 & 20.6 \\
\hline $\begin{array}{c}\text { Opening } \\
\text { gap }\end{array}$ & $\mathrm{U} 1$ & 26.7 & 26.8 & 27.3 & 27.64 & 28.1 & 29.12 & 30.54 & 31.52 & 33.9 & 36.4 \\
\hline $\begin{array}{c}\text { length, } \\
\text { mm }\end{array}$ & $\mathrm{N}$ & 32.8 & 33 & 33.52 & 34.08 & 34.48 & 35.24 & 36.52 & 37.82 & 40.34 & 43.06 \\
\hline
\end{tabular}

Here, $\mathrm{U} 1$ means used ring 1; $\mathrm{U} 2$ is used ring 2, and $\mathrm{N}$ is the new unused ring.

Based on the collected data, the relationships between the opening gap length and the applied load are shown in Figure 21 for the used and new seal rings. It is clearly seen that the used seal rings are less stiff compared with the new ring because the variation of the opening gap length of used seal rings is larger than the new seal ring. The stiffness change with use also can be explained by the reduced used seal ring width due to wear. The used seal rings were taken from the same failed turbine, which explains why both used seal rings have the same stiffness, and supports the argument that the change in the seal ring stiffness was due to wear.

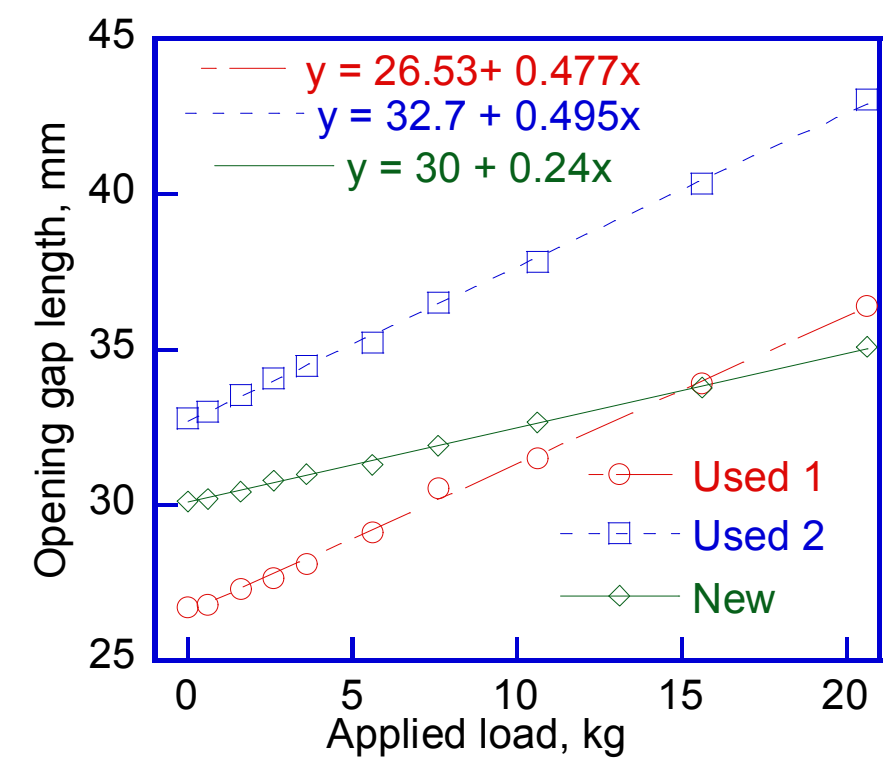

Figure 21. Relationships between the opening gap length and the externally applied load. 


\subsection{Elastic Modulus, Hardness and Bulk Chemical Composition}

After grinding and polishing the specimens, the hardness and elastic modulus of the new and used seal ring materials were measured by nanoindentation. The results are presented in Tables 4 and 5 .

As a result, the elastic modulus and hardness of the new seal ring material are higher than the used one. The seal ring working environment is complex. The working pressure is $4.5-5.5$ $\mathrm{MPa}$ and the working temperature is $523-573 \mathrm{~K}$. Thus, these evidences show that temperature and stress as external conditions are responsibile for the failure of the seal ring.

Additionally, high temperature environment and working medium will lead to variable of the microstructure. Therefore, hardness decrease with use can be explained by the recovery and recrystallization processes of the ring material. Bulk chemical composition of the new and used rings is listed in Table 6 . There are no significant differences in chemical composition, so the primary cause of the reduction of the elastic modulus and hardness can be explained by the microstructure changes with use due to recovery, recrystallization and secondary phases precipitation.

Moreover, during the nanoindentation test, the four points on the surface were randomly selected. The hardness of FCC nickel material on the nano-scale is significantly affected by crystallographic surfaces with different orientations. The hardness value of the (100) substrate is the greatest and the hardness values of (111) substrate is the smallest. The reason is because the (100) substrate has only one slip angle of the $\{111\}$ sliding planes and the (111) substrate has three slip angles, respectively, during nanoindentation, resulting in the (100) substrate having a lower ability for plastic deformation, ultimately causing a higher hardness value. 
The used seal ring experienced plastic deformation, so that strain energy was stored. The tip force will decrease when the indentation depths increase, because the strain energy of used seal ring and force exerted by the tip will be released by the dislocation sliding along the $\{111\}$ plane. Furthermore, there are lots of precipitates in the grain boundaries. If the indents test near this part, the atoms around the tip reveal the repulsive stresses and the atomic normal stresses increase with the indentation depth increase. Both of them also might lead to lower hardness values. However, the experimental values for hardness are still in a reasonable range [31].

Table 4. New seal ring mechanical properties.

\begin{tabular}{|c|c|c|}
\hline Test & Elastic modulus, GPa $(\mathbf{( 1 )}$ & Hardness, GPa $(\mathbf{( 0 . 1 )}$ \\
\hline 1 & 306 & 4.9 \\
\hline 2 & 305 & 5.2 \\
\hline 3 & 323 & 5.1 \\
\hline 4 & 329 & 5.1 \\
\hline Average & 316 & 5.1 \\
\hline
\end{tabular}

Table 5. Used seal ring mechanical properties.

\begin{tabular}{|c|c|c|}
\hline Test & Elastic modulus, GPa $(\mathbf{\pm 1})$ & Hardness, GPa $(\mathbf{\pm 0 . 1})$ \\
\hline 1 & 265 & 3.3 \\
\hline 2 & 303 & 3.3 \\
\hline 3 & 267 & 3.1 \\
\hline 4 & 271 & 3 \\
\hline Average & 277 & 3.2 \\
\hline
\end{tabular}

Table 6. Main elements composition of the used and new seal rings.

\begin{tabular}{|c|c|c|c|c|c|c|c|}
\hline \multicolumn{2}{|c|}{ Elements } & $\mathrm{Fe}$ & $\mathrm{Cr}$ & $\mathrm{Ni}$ & $\mathrm{Mo}$ & $\mathrm{Cu}$ & $\mathrm{Ti}$ \\
\hline \multirow{2}{*}{$\begin{array}{c}\text { Composition, } \\
\text { wt.\% }\end{array}$} & Used & 54.5 & 15.27 & 24.64 & 1.52 & 0.69 & 2.85 \\
\cline { 2 - 8 } & New & 53.82 & 14.86 & 25.36 & 1.56 & 0.61 & 2.83 \\
\hline
\end{tabular}

\subsection{Metallographic, SEM and EDS Analyses}

Different surfaces of the seal ring were observed using optical microscopy and there were no obvious differences between the cross section, front and back surfaces, thus the back surface 
was used for microstructure comparisons. Figure 22 shows optical images of the new and used rings back surface. Austenite grains can be clearly seen in both used and new rings, however, the new ring grain size in Fig. 22 (a) is larger than the used ring in Fig. 22 (b). The new seal ring average grain size is about $206 \mu \mathrm{m}$, while the average grain size of the used seal ring is $62 \mu \mathrm{m}$. The used seal ring has undergone serious grain refinement. Additionally, many precipitates can be observed at grain boundaries of the used ring in Fig. 22 (b), opposite to the new ring in Fig. 22 (a). Thus, it is reasonable to assume that the secondary phase precipitation occurred in the used rings along the grain boundaries, which is not present in the new ring. Compared with the new seal ring, the used seal ring material had many precipitates and more serious erosion of the grain boundaries due to the etching solution.
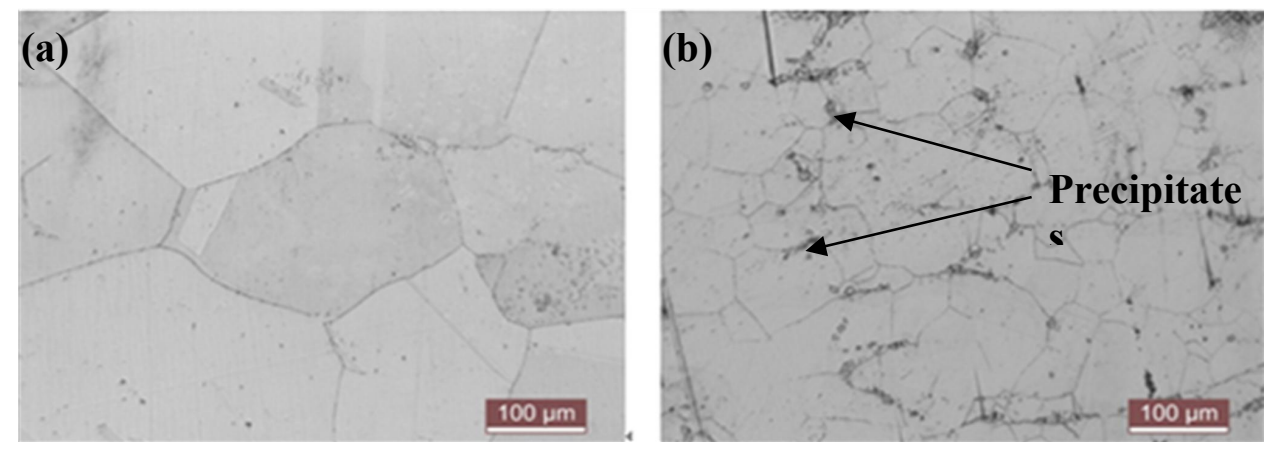

Figure 22. Optical microscopy images of: (a) New seal ring back surface; (b) Used seal ring back surface showing precipitates along the grain boundaries.

Regardless of the fact that the used steel ring grain size is significantly smaller than the new one, the used ring has lower hardness. It is reasonable to assume that the secondary phase precipitation occurred in the used rings, accompanied by high dislocation density due to use. While the operating temperature of $523-573 \mathrm{~K}$ is below steel recrystallization temperature [32], recovery affected the performance of the used seal ring. Because of the low operating temperature, atomic diffusion capacity is weak. However, the ring is also mechanically loaded during use, providing additional energy for dynamic recovery and recrystallization [33]. 
High nickel alloy steel material of the seal ring is austenitic steel with low stacking fault energy. Dynamic recovery is very slow because it is difficult for extended dislocations to escape from the dislocation network, and it is also difficult for dislocations to move by sliding and climbing. At the same time, high dislocation density in the sub-structure and the remaining energy can cause recrystallization. Thus, dynamic recovery and dynamic recrystallization soften the used seal ring material, as demonstrated by the hardness measurements [34-35].

SEM micrograph, elemental maps and EDS analysis of the used seal ring are presented in Figure 23 to analyze the precipitates composition. Combined elements map is shown in Fig. 23(b), while individual elemental maps for Si, Fe, Cr, Ni, Ti and Mo are shown in Fig. 23(c). From the individual element maps it is clear that the precipitates composition is complex. EDS analysis results of points 1 and 2 in Fig. 23(a) are listed in Table 7, showing increased Ti and Mo concentrations. Moreover, the content of $\mathrm{Ni}, \mathrm{Si}$, Ti and Mo is higher in the precipitates regions along the grain boundaries, as seen in the combined and individual element maps in Fig. 23(b) and (c).

Table 7. Used seal ring precipitate composition elements analysis by EDS.

\begin{tabular}{|c|c|c|c|c|c|}
\hline Point 1 & $\begin{array}{c}\text { Conc. } \\
\text { [wt.\%] }\end{array}$ & $\begin{array}{c}\text { Conc. } \\
\text { [at.\%] }\end{array}$ & Point 2 & $\begin{array}{c}\text { Conc. } \\
\text { [wt.\%] }\end{array}$ & $\begin{array}{c}\text { Conc. } \\
\text { [at.\%] }\end{array}$ \\
\hline $\mathrm{C}$ & 11.74 & 36.32 & $\mathrm{C}$ & 23.92 & 59.1 \\
\hline $\mathrm{O}$ & 3.01 & 6.98 & $\mathrm{Si}$ & 1.42 & 1.5 \\
\hline $\mathrm{Si}$ & 3.02 & 4 & $\mathrm{Cl}$ & 0.46 & 0.39 \\
\hline $\mathrm{Ti}$ & 11.24 & 8.72 & $\mathrm{Ti}$ & 2.67 & 1.65 \\
\hline $\mathrm{Cr}$ & 15.54 & 11.1 & $\mathrm{Cr}$ & 11.1 & 6.34 \\
\hline $\mathrm{Fe}$ & 22.86 & 15.21 & $\mathrm{Fe}$ & 38.43 & 20.42 \\
\hline $\mathrm{Ni}$ & 20.58 & 13.03 & $\mathrm{Ni}$ & 19.38 & 9.8 \\
\hline $\mathrm{Mo}$ & 12 & 4.65 & $\mathrm{Mo}$ & 2.61 & 0.81 \\
\hline
\end{tabular}



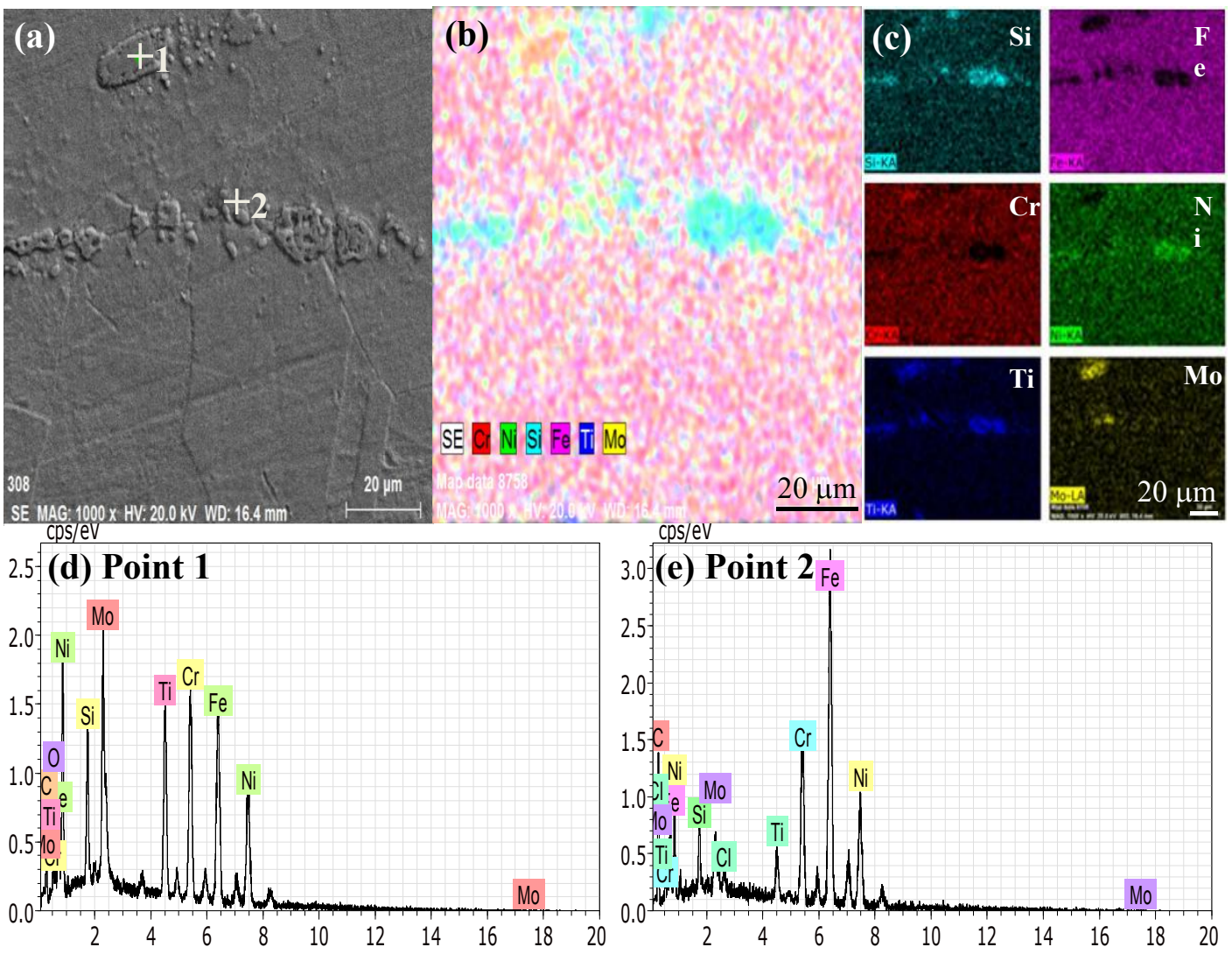

Figure 23. (a) SEM micrograph; (b) Combined elemental map; (c) Individual elemental maps; (d) EDS spectra of point 1 and (e) EDS spectra of point 2 of the used seal ring labeled in (a).

Unlike the used ring, no precipitation along the grain boundaries was observed in the new ring material. Table 8 lists elemental composition results of the new ring material obtained by EDS.

Table 8. New seal ring precipitate composition elements analysis by EDS.

\begin{tabular}{|c|c|c|c|c|c|}
\hline Point 1 & $\begin{array}{c}\text { Conc. } \\
\text { [wt.\%] }\end{array}$ & $\begin{array}{c}\text { Conc. } \\
\text { [at.\%] }\end{array}$ & Point 2 & $\begin{array}{c}\text { Conc. } \\
\text { [wt.\%] }\end{array}$ & $\begin{array}{c}\text { Conc. } \\
\text { [at.\%] }\end{array}$ \\
\hline $\mathrm{C}$ & 6.34 & 24.22 & $\mathrm{C}$ & 8.63 & 31.14 \\
\hline $\mathrm{Al}$ & 0.83 & 1.42 & $\mathrm{Al}$ & 0.68 & 1.1 \\
\hline $\mathrm{Ti}$ & 2.71 & 2.6 & $\mathrm{Cl}$ & 0.44 & 0.54 \\
\hline $\mathrm{Cr}$ & 13.57 & 11.97 & $\mathrm{Ti}$ & 5.12 & 4.63 \\
\hline $\mathrm{Fe}$ & 6.66 & 5.47 & $\mathrm{Cr}$ & 12.04 & 10.04 \\
\hline $\mathrm{Ni}$ & 68.89 & 53.84 & $\mathrm{Fe}$ & 6.06 & 4.7 \\
\hline $\mathrm{Nb}$ & 1 & 0.49 & $\mathrm{Ni}$ & 60.85 & 44.95 \\
\hline & & & $\mathrm{Nb}$ & 6.17 & 2.88 \\
\hline
\end{tabular}


Compared with the used seal ring, there is much more Ni in the new seal ring steel. At the same time, $\mathrm{Nb}$ and $\mathrm{Al}$ can be found in the used seal ring material, which were not present in the new ring. Adding alloying elements, such as $\mathrm{Al}, \mathrm{Ti}$ and $\mathrm{Nb}$, to high nickel alloy steel, results in precipitation strengthening, producing secondary phase, while controlling over-saturated solid solution [36]. This is mainly based on the $\mathrm{Ni}_{3} \mathrm{Al}$ intermetallic compound, called the $\gamma$ phase. $\mathrm{Al}$ hardens high nickel alloy steel. However, the $\gamma$ phase has higher solid solubility for $\mathrm{Ti}$ and $\mathrm{Nb}$. Thus, adding them can reduce the effects of Al. Moreover, $\mathrm{Nb}$ can decrease the ageing rate $[37-40]$.

The seal ring maximum working temperature is around $573 \mathrm{~K}$, which is not high enough to cause complete recrystallization. Thus, the mechanical stress of 4.5-5.5 $\mathrm{MPa}$ during operation needs to be considered. Combination of stress and temperature causes the seal ring failure. As a final step to identify the cause of the ring failure, in-situ tensile test was conducted in SEM with new and used ring materials.

\subsection{In-situ SEM Tensile Test}

Identical tensile specimens, $22 \mathrm{~mm}$ long and $1 \mathrm{~mm}$ thick were cut from the used and new seal ring materials. During the tensile test, the specimens of seal ring transited from elastic to plastic deformation. Dislocation, fine-grain and precipitation could contribute to the rise of seal ring's yield strength [41]. In-situ tensile test and observation of the specimen surface proved that the grain boundaries are the weakest areas of the used seal ring material. Surface morphology of the used and new ring materials is shown in Figure 24. 

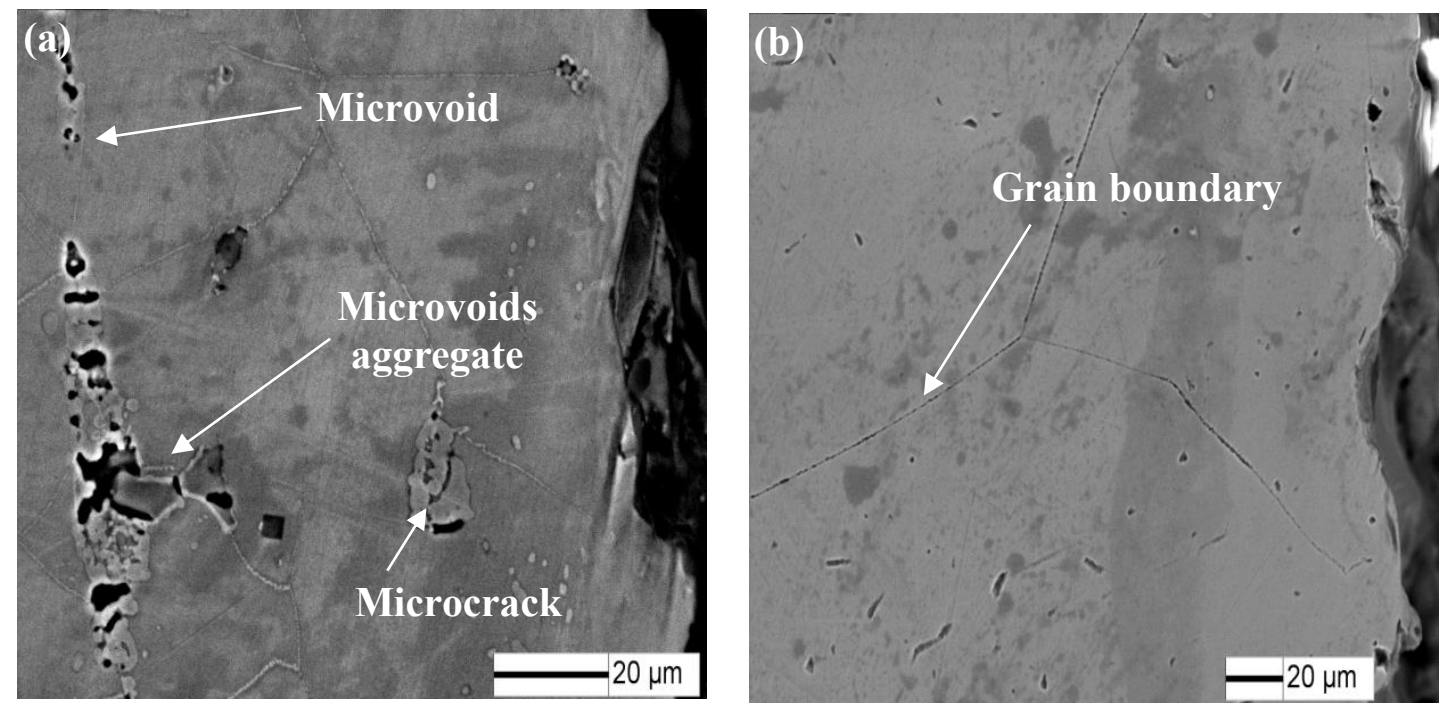

Figure 24. SEM images of: (a) Used seal ring and (b) New seal ring obtained during in-situ tensile test.

Microvoids first appeared at the precipitates along the grain boundaries of the used ring material. Then microvoids aggregated, finally forming microcracks in Fig. 24(a). No such phenomena were observed in the new seal ring material in Fig. 24(b), where only grain boundaries can be seen without voids or cracks during the in-situ SEM tensile test. Therefore, precipitates are the main reason causing the seal ring failure and the grain boundaries are the weakest areas in the used seal ring. Microvoids aggregation eventually caused the formation of macroscopic cracks, leading to seal ring material failure. 


\section{CHAPTER 4: CONCLUSION AND FUTURE WORK}

Seal ring complex work environment analysis, including temperature and stress, shows that the used seal ring lost its ability to form a complete seal, resulting in leakage and failure. Used seal ring obviously appears plastically deformed. Dynamic recrystallization of the used ring material caused grain refinement, which was reflected by reduced hardness. Moreover, creep aging also caused the stiffness of the used seal ring decrease and failure due to long-term service. At the same time, precipitates appeared along the grain boundaries [42], leading to ring failure by forming cracks along the grain boundaries. The effects of precipitation contributed to grain refinement. Ti and Mo precipitates reduced the stacking fault energy, promoting dynamic recrystallization.

In the future, the nuclear power must be an important part in the modern industry. Thus, the security application of nuclear power is an inevitable research focus. In this project, the failure of seal ring had seriously impacted on the normal operation of the steam turbine system. The used seal ring had been tested through SEM and in-situ tensile test. The circumstances of dislocation and corrsive behaviour also should be observed. Furthermore, the cosome suggestions need to be given in order to avoid the seal ring failure and extend its service life. 


\section{REFERENCES}

[1] N. Oishi, M. Tanaka, Periodic thermal deformation of large seal ring. JSME International Journal. Ser. C, Dynamics, Control, Robotics, Design and Manufacturing 39(2) (1996) 347-355.

[2] Forged heat-resisting superalloy discs, YB/T 5351-2006 Chinese standard, 2006.

[3] Bloch, H. P. Singh, M. Steam Turbines: Design, Application, and Re-Rating. McGraw Hill Professional. (2008).

[4] Li, Y. Wang, C. Study on the Effect of Regenerative System on Power Type Relative Internal Efficiency of Nuclear Steam Turbine. Energy Procedia, 17 (2012) 906-912.

[5] Okuno, K. Iwasaki, Y. Murata, Y. Hirano, T. Murakami, I. Ohashi, S. U.S. Patent Application No. 13/955 (2013) 760.

[6] Soares, C. Gas turbines: a handbook of air, land and sea applications. ButterworthHeinemann. (2011).

[7] Ma, M. T. Sherrington, I. Smith, E. H. Grice, N. Development of a detailed model for piston-ring lubrication in IC engines with circular and non-circular cylinder bores. Tribology International. 30(11) (1997) 779-788.

[8] Kim, G. H. Lee, Y. S. Yang, H. L. A new design concept of metal O-ring seal for long-term performance. Vacuum. 123 (2016) 54-61.

[9] Jan, S. U.S. Patent No. 6,283,479. Washington, DC: U.S. Patent and Trademark Office. (2001).

[10] Higa, Y. Tomita, Y. Advance Materials and Modeling of Mechanical Behavior. Fleming Printing Ltd. 3 (1999) 1095-1099. 
[11] Shibkov, A. A. Zolotov, A. E. Mikhlik, D. V. Zheltov, M. A. Shuklinov, A. V. Nucleation and multiplication of Savart-Masson bands in an 5456 alloy. Russian Metallurgy (Metally), 2010(10) (2010). 874-880.

[12] Ivanova, V. S. Synergetics: strength and fracture of metallic materials. CAMBRIDGE INTERNATIONAL SCIENCE PUBLISHING, 7 Meadow Walk, Great Abington, CAMBRIDGE CB 16 AZ, England, 1995140.

[13] Pázmán, J. Bereczki, P. Vero, B. Kardos, I. Feher, J. The Effect of the Severe Plastic Deformation on the Artificial Aging of the AlMgSil Alloy. In Materials Science Forum Trans Tech Publications Ltd. (2015, February) (Vol. 812, p. 239).

[14] Van Zyl, I. Yadroitsava, I. Yadroitsev, I. Residual stress in Ti6Al4V objects produced by Direct Metal Laser Sintering. South African Journal of Industrial Engineering, 27(4) (2016) 134-141.

[15] Saka, H. Factors affecting the dissociation width of dissociated dislocations in FCC metals and alloys. Journal of materials science, 51(1) (2016) 405-424.

[16] Pang, W. W. Zhang, G. C. Zhao, X. G. Zhang, P. Dependence of dislocation creation on tensile orientation in face-centered-cubic ductile metals under high strain rate loading. Journal of Applied Physics, 118(12) (2015) 124301.

[17] Gorski, C. A. Fantle, M. S. Stable mineral recrystallization in low temperature aqueous systems: A critical review. Geochimica et Cosmochimica Acta, 198 (2017) 439-465.

[18] ASM Handbook. Vol.11: Failure Analysis and Prevention (10th Ed.). ASM International, 2002. P2884.

[19] Ashby, M. F. Gandhi, C. Taplin, D. M. R. Overview No. 3 Fracture-mechanism maps and their construction for fcc metals and alloys.Acta metallurgica, 27(5) (1979) 699-729.

[20] Kumar, M. Singh, I. V. Mishra, B. K. Ahmad, S. Rao, A. V. Kumar, V. A Modified Theta Projection Model for Creep Behavior of Metals and Alloys. Journal of Materials Engineering and Performance. 25(9) (2016) 3985-3992. 
[21] Barbera, D. Chen, H. Liu, Y. Creep-fatigue behaviour of aluminum alloy-based metal matrix composite. International Journal of Pressure Vessels and Piping. 139 (2016) 159-172.

[22] Hou, Y. Lei, D. Li, S. Yang, W. Li, C. Q. Experimental investigation on corrosion effect on mechanical properties of buried metal pipes. International Journal of Corrosion, 2016.

[23] Chen, Y. Zhang, H. Zhang, J. Li, X. Zhou, J. Failure analysis of high strength pipeline with single and multiple corrosions. Materials \& Design, 67 (2015) 552-557.

[24] Taiwade, R. V. Patil, A. P. Patre, S. J. Dayal, R. K. Effect of solution annealing on susceptibility to intercrystalline corrosion of stainless steel with $20 \% \mathrm{Cr}$ and $8 \% \mathrm{Ni}$. Journal of materials engineering and performance, 22(6) (2013) 1716-1728.

[25] Majumdar, S. Bakhtiari, S. Kasza, K. Park, J. Y. Validation of Failure and Leak Rate Correlations for Stress Corrosion Cracks in Steam Generator Tubes. In NUREG/CR-6774. US Nuclear Regulatory Commission Washington, DC. (2002).

[26] Antsupov, A. V. Rusanov, V. A. Antsupova, I. A. Modeling of Wear-Out Failures and Service Life Improvement of Sealing Units. Procedia Engineering, 150 (2016). 416-425.

[27] M.B. Lin, K. Gao, C.J. Wang, A.A. Volinsky, Failure analysis of the oil transport spiral welded pipe. Engineering Failure Analysis 25 (2012) 169-174.

[28] Marteau, J. Mazeran, P. E. Bouvier, S. Bigerelle, M. Zero-Point Correction Method for Nanoindentation Tests to Accurately Quantify Hardness and Indentation Size Effect. Strain 48(6) (2012) 491-497.

[29] Leng, Y. Materials characterization: introduction to microscopic and spectroscopic methods. John Wiley \& Sons (2009).

[30] Uvarov, V. L. A. D. I. M. I. R. Popov, I. Metrological characterization of X-ray diffraction methods for determination of crystallite size in nano-scale materials. Materials characterization, 58(10) (2007) 883-891. 
[31] C. H. Chen, The nanoindentation responses of nickel surfaces with different crystal orientations. Molecular Simulation. 33 (11) (2007) 905-917.

[32] Z. Larouk, H. Bouhalais, Recrystallization behavior of a low carbon steel wire, Physics Procedia 2(3) (2009) 1223-1229.

[33] E. Cerri, P. Leo, Influence of severe plastic deformation on aging of Al-Mg-Si alloys. Materials Science and Engineering: A 410 (2005) 226-229.

[34] P. Zhang, C. Yi, G. Chen, H. Qin, C. Wang, Constitutive model based on dynamic recrystallization behavior during thermal deformation of a nickel-based superalloy. Metals 6(7) (2016) 161 doi:10.3390/met6070161.

[35] C. Sun, Y. Xiang, Q. Zhou, D.J. Politis, Z. Sun, M. Wang, Dynamic recrystallization and hot workability of 316LN stainless steel. Metals 6(7) (2016) 152 doi:10.3390/met6070152.

[36] B.H. Kear, D.P. Pope, Role of Refractory elements in strengthening of $\gamma^{\prime}$ and $\gamma$ precipitation hardened nickel-base superalloys, Superalloys Supercomposites Superceramics, Academic Press (1989) 545-582.

[37] L. Luo, Y. Huang, S. Weng, F. Z. Xuan, Mechanism-related modelling of pit evaluation in the $\mathrm{CrNiMoV}$ steel in simulated environment of low pressure nuclear steam turbine. Materials \& Design 105 (2016) 240-250.

[38] M.N. Ahmadabadi, H. Shirazi, H. Ghasemi-Nanesa, S. H. Nedjad, B. Poorganji, T. Furuhara, Role of severe plastic deformation on the formation of nanograins and nano-sized precipitates in Fe-Ni-Mn steel. Materials \& Design 32(6) (2011) 3526-3531.

[39] B.Piekarski, Effect of $\mathrm{Nb}$ and $\mathrm{Ti}$ additions on microstructure, and identification of precipitates in stabilized Ni-Cr cast austenitic steels. Materials Characterization 47(3) (2001) 181-186.

[40] S.L. Zhu, H.Z. Cao, J.S. Ye, W.H. Hu, G.Q. Zheng, Dynamic recrystallization behavior of medium carbon $\mathrm{Cr}-\mathrm{Ni}-\mathrm{Mo}-\mathrm{Nb}$ steel during hot deformation. Journal of Iron and Steel Research International 22(3) (2015) 264-271. 
[41] Bouaziz, H. Brinza, O. Haddar, N. Gasperini, M. Feki, M. In-situ SEM study of crack initiation, propagation and interfacial debonding of Ni-P coating during tensile tests: Heat treatment effect. Materials Characterization, 123 (2017) 106-114.

[42] G. Sun, Q. Ma, In-situ observation of dark phase precipitation during heating and soaking process of a high nickel steel. Journal of Wuhan University of Technology-Mater. Sci. Ed.30(1) (2015) 152-155. 


\begin{abstract}
ABOUT THE AUTHOR
Wenbo Wang graduated from the Lanzhou University of Technology with the bachelor degree of welding technology and engineering. He earned the certification of International Welding Engineer (I.W.E) during his undergraduate study. Now he is pursuing master degree of material science and engineering at the University of South Florida.
\end{abstract}

